\title{
EXTREMAL LENGTH ESTIMATES AND PRODUCT REGIONS IN TEICHMÜLLER SPACE
}

\author{
YAIR N. MINSKY \\ September 1, 1994
}

\begin{abstract}
We study the Teichmüller metric on the Teichmüller space of a surface of finite type, in regions where the injectivity radius of the surface is small. The main result is that in such regions the Teichmüller metric is approximated up to bounded additive distortion by the sup metric on a product of lower dimensional spaces. The main technical tool in the proof is the use of estimates of extremal lengths of curves in a surface based on the geometry of their hyperbolic geodesic representatives.
\end{abstract}

\section{$\S 1$. Introduction}

There is a longstanding but imperfect analogy between the geometry of the Teichmüller space $\mathcal{T}(S)$ of a surface and that of a complete, negatively curved space. For example, $\mathcal{T}(S)$ admits a boundary at infinity similar to that of hyperbolic space (See e.g. $[8,20]$ ), and the Teichmüller geodesic flow on its quotient, the moduli space, is ergodic (see [19]). This paper studies one of the strong ways in which this analogy fails, namely the existence of large regions in the space which are closely approximated by products of lower-dimensional metric spaces.

There are several natural metrics on $\mathcal{T}(S)$; we will consider throughout only the Teichmüller metric (§2.5). The first indication of positively curved behavior of the Teichmüller metric came from Masur [17], in which examples were given of geodesic rays with a common basepoint, which remain a bounded distance apart for all time. This contradicts non-positive curvature on small scales. More recently, Masur and Wolf [21] have given examples of geodesic triangles which fail, arbitrarily badly, the "thin triangle" condition for hyperbolicity in the sense of Gromov and Cannon $[5,6,11]$; this implies that the large-scale behavior of the metric is not negatively curved. The main result of this paper describes regions of $\mathcal{T}(S)$ where the large scale behavior exhibits some characteristics of positive curvature.

The main theorem can be summarized as follows (a complete statement appears in $\S 6$ ). Let $\mathcal{T}(S)$ be the Teichmüller space of a surface $S$ of finite type, endowed with the Teichmüller metric. Let $\gamma=\gamma_{1}, \ldots, \gamma_{k}$ be a system of disjoint, homotopically

This work was partially supported by an NSF postdoctoral fellowship. 
distinct simple closed curves on $S$, and let $\operatorname{Thin}_{\epsilon}(S, \gamma)$ denote the set of $\sigma \in \mathcal{T}(S)$ for which $\ell_{\sigma}\left(\left[\gamma_{i}\right]\right) \leq \epsilon$ for all $i$, where $\ell_{\sigma}\left(\left[\gamma_{i}\right]\right)$ denotes hyperbolic length of the $\sigma$-geodesic representative of $\gamma_{i}$. Let $X_{\gamma}$ denote the product space $\mathcal{T}(S \backslash \gamma) \times \mathbf{H}_{1} \times \cdots \times \mathbf{H}_{k}$, where $S \backslash \gamma$ is considered as a punctured surface, and each $\mathbf{H}_{i}$ is a copy of the hyperbolic plane. Endow $X_{\gamma}$ with the sup metric, $d_{X}=\max \left\{d_{\mathcal{T}(S \backslash \gamma)}, d_{\mathbf{H}_{1}}, \ldots, d_{\mathbf{H}_{k}}\right\}$, of the metrics on the factors.

Theorem 6.1 The Fenchel-Nielsen coordinates on $\mathcal{T}(S)$ give rise to a natural homeomorphism $\Pi: \mathcal{T}(S) \rightarrow X_{\gamma}$, and for $\epsilon$ sufficiently small this homeomorphism restricted to $\operatorname{Thin}_{\epsilon}(S, \gamma)$ distorts distances by a bounded additive amount. That is,

$$
\left|d_{\mathcal{T}(S)}(\sigma, \tau)-d_{X}(\Pi(\sigma), \Pi(\tau))\right| \leq c
$$

For $\sigma, \tau \in \operatorname{Thin}_{\epsilon}(S, \gamma)$, and $c=c(\chi(S), \epsilon)$.

Note in particular that the distortion of $\Pi$ is additive and not multiplicative, as is more commonly the case with quasi-isometries. For this reason it is significant that we endow $X_{\gamma}$ with the sup metric, and not the $L_{2}$ metric; these are equivalent up to bounded multiplicative, but not additive, distortion. A product of spaces with the sup metric exhibits not just non-negative, but actually positive curvature characteristics in the large. See $\S 7$ for a fuller discussion of this point.

The idea of decomposing Teichmüller space as a product using length and twist parameters of simple closed curves dates back to Fenchel-Nielsen [9] (see Abikoff [1, Chap. II] and Fricke-Klein [10]). Such decompositions have particular significance when the curves in question are short, and this has been used by several authors to study the degeneration of Riemann surfaces towards "noded" surfaces, in which a short curve has been replaced by two punctures. From a complex-analytic and algebraic-geometric point of view, such degenerations have been investigated by e.g. Bers [4], Earle-Marden [7], Kra [13], Maskit [14, 15], Masur [18] and Wolpert $[26,27,25]$. In all these cases, the emphasis is on careful local analysis, concentrating on the complex structure of the moduli space in a neighborhood of a noded surface, or on differential geometric structure such as the Weil-Petersson metric in the case of $[18,25]$, or a precise expression for the hyperbolic metric on the surface in [27]. By contrast, our approach ignores most local analytical details and concentrates on large-scale estimates in the Teichmüller space.

Extremal length estimates. The estimates of Teichmüller distance necessary for theorem 6.1 are obtained using Kerckhoff's theorem (see §2.5), which relates Teichmüller distance to ratios of extremal lengths. Therefore our main tool is a theorem that allows us to estimate extremal lengths of arbitrary curves on a surface, in terms of the behavior of their hyperbolic geodesic representatives.

The relationship between hyperbolic length $\ell_{\sigma}$ and extremal length $\lambda_{\sigma}$ (see §2.3) of curves on a Riemann surface $(S, \sigma)$ is not completely straightforward. For very short curves, $\ell_{\sigma}$ and $\lambda_{\sigma}$ are nearly proportional. For long curves, the relation depends on the geometry of the surface. In particular an upper bound for $\lambda_{\sigma}$ is given by $\lambda_{\sigma} \leq(1 / 2) \ell_{\sigma} e^{\ell_{\sigma} / 2}$ (see Maskit [16]), and this is close to sharp in general. But on the other hand $\lambda_{\sigma} \geq \ell_{\sigma}^{2} / 2 \pi|\chi(S)|$ is the general lower bound that comes from the 
definition of extremal length, and for any fixed conformal structure $\sigma$ one can show (see e.g. [23] and lemma 4.3) that it is the lower bound which estimates $\lambda_{\sigma}$ to within a bounded factor.

Theorem 5.1 gives an estimate for $\lambda_{\sigma}$ which is correct, up to a bounded factor that depends only on the topological type of the surface, for all cases. The main observation is that, after decomposing a Riemann surface $(S, \sigma)$ into thick and thin parts, or what we call here an $\left(\epsilon_{0}, \epsilon_{1}\right)$ collar decomposition (see $\S 2.4$ ), each component of the decomposition contributes individually to the extremal length of a curve $\beta$ in $S$. The contribution $\lambda_{P, \sigma}(\beta)$ of a component $P$ of the thick part (see definition (4.2) and lemma 4.3) is approximately $\ell_{\sigma}^{2}\left(\beta^{\sigma} \cap P\right)$, where $\beta^{\sigma}$ is the $\sigma$-geodesic representing $\beta$. A component $A$ of the thin part, which is an annulus, contributes a quantity $\lambda_{A, \sigma}(\beta)$ which depends on the modulus of $A$ and the amount of "twisting" that $\beta$ does in $A$ (see $\S 3$ and definition (4.3)). The theorem then states:

Theorem 5.1 Let $S$ be a surface of finite type with a standard hyperbolic metric $\sigma$ in which boundary lengths are at most $\ell_{0}$, and let $\mathcal{Q}$ be the set of components of the $\left(\epsilon_{0}, \epsilon_{1}\right)$ collar decomposition. Then, for any curve system $\beta$ in $S$, the extremal length $\lambda_{S, \sigma}(\beta)$ is approximated by

$$
\max _{Q \in \mathcal{Q}} \lambda_{Q, \sigma}(\beta)
$$

up to multiplicative factor depending only on $\epsilon_{0}, \epsilon_{1}, \ell_{0}$ and the topological type of $S$.

In specific cases this theorem recovers the above exponential and quadratic bounds from [16] and [23].

Proofs. The proof of theorem 5.1 is essentially a long exercise in elementary hyperbolic geometry. Given a homotopy class of curves $\beta$ in $S$ we must arrange it in a sufficiently well-spaced way so as to obtain an embedded annulus which gives us an upper bound for extremal length (lower bounds are easier). The part of the argument which is not straightforward has to do with finding an appropriate metric, conformally equivalent to the hyperbolic metric, in which to make the spacing construction. The scaling factors, which vary among components of the thick-thin decomposition, must depend on the way in which $\beta$ is distributed on $S$. The construction of this metric is given in lemma 5.2 and the discussion that follows it. (Note that the metric we obtain is in some way mimicking the actual extremal metric, which is given by a quadratic differential. However our construction is explicitly related to the hyperbolic characteristics of $\beta$, which makes it more suited to our purposes.)

The proof of theorem 6.1 is an almost direct application of theorem 5.1 and Kerckhoff's theorem 2.1, except for one point. In the passage from a component of $S \backslash \gamma$ with (short) boundary to a punctured surface, we must at some point compare extremal length ratios $\lambda_{\sigma} / \lambda_{\tau}$ for properly embedded arcs with endpoints on the boundary to similar ratios for simple closed curves in the interior. That the suprema of these ratios are within a bounded factor of each other is shown in lemma 6.2. The main trick in this lemma is start with a (possibly non-closed) curve 
$\beta$ that maximizes the reciprocal ratio $\lambda_{\tau} / \lambda_{\sigma}$, and then find a closed curve which is sufficiently close to being "orthogonal" to $\beta$.

A different approach to the proof of theorem 6.1 than the one we have taken might involve estimating Teichmüller distances directly by constructing appropriate quasiconformal maps. One might hope that, since a short curve corresponds to a high-modulus annulus in the surface, a quasi-conformal map on the complement of this annulus might be "spliced" across the annulus in a way that takes the appropriate modulus and twist parameters into account, and thus the quasi-conformal dilatation of the spliced map would be estimated by the dilatations of the component maps (and the modulus and twisting information in the annulus). It is therefore of interest that, in our experience, this approach actually runs into quite serious difficulties, since "splicing" quasi-conformal maps is, in general, not very easily done without great loss of control over dilatation. In particular, estimates of dilatation of such spliced maps tend to be, at best, polynomially related to the dilatations of the component maps, and not linearly as we would need for theorem 6.1. It is for this reason that we have adopted the extremal length approach, which circumvents these problems.

Summary of contents. In section 2 we set our notation and recall some wellknown facts about conformal structures on surfaces, curves and their lengths, and Teichmüller distance. The only proof we give is an elementary one for lemma 2.2.

In section 3 we define twisting numbers, which are a convenient way to measure how many times one geodesic twists around another in a hyperbolic surface, and in particular inside a Margulis annulus. We prove a few elementary facts about them, and relate them to Fenchel-Nielsen twist parameters in lemma 3.5.

Section 4 gives some well-known estimates of extremal lengths, in particular an estimate in terms of hyperbolic length in a component of the thick part (lemma 4.3). Lemma 4.4 describes briefly how to space a curve evenly in a component of the thick part, and this will be used in $\S 5$. At the end of the section we define the annulus contribution $\lambda_{A, \sigma}$ to extremal length, in terms of twisting numbers.

Sections 5 and 6 give the proof of theorems 5.1, and 6.1, respectively. Section 7 gives a brief discussion of the geometric consequences of theorem 6.1.

Acknowledgements. The author is grateful to the Long Island Rail Road for its generosity in providing space and time for contemplation.

\section{$\S 2$. Definitions and background}

$\S 2.1$. Metrics and conformal structures on surfaces. Let $S$ be a smooth orientable surface of finite topological type, by which we mean that $S$ is homeomorphic to a connected, compact surface (possibly with boundary) minus a finite set, which we call punctures or cusps.

By conformal structure on $S$ we shall mean a conformal structure on the interior, for which each puncture has a neighborhood conformally equivalent to a punctured disk, and each boundary component has a neighborhood conformally equivalent to an annulus of finite modulus. Let $\mathcal{T}(S)$ denote the Teichmüller space of marked 
conformal structures on $S$ considered up to conformal homeomorphisms homotopic to the identity (see [1]). If $S$ has no boundary (but possibly punctures) then $\mathcal{T}(S)$ is the space of analytically finite marked conformal structures.

A conformal structure also determines a class of metrics on the interior, all those that can be described in a local conformal coordinate as a positive function times the Euclidean metric. In this paper we will use metrics which are smooth or mildly singular (non-differentiable on some curves), and which have a completion to the boundary of $S$. The conformal class of a metric $\sigma$ will be denoted $[\sigma]$.

Call $S$ hyperbolic if its Euler characteristic is negative, which implies that it admits a complete finite-volume hyperbolic metric in which the boundary is geodesic. We will call such a hyperbolic metric on $S$ standard. We say a Euclidean metric on an annulus is standard if the annulus can be obtained isometrically from a Euclidean rectangle by identifying a pair of opposite edges.

$\S$ 2.2. Curve systems. A curve system on $S$ is a disjoint union of homotopically non-trivial properly embedded simple arcs and closed curves (for an arc, non-trivial means not deformable into $\partial S$ with endpoints fixed, and for a closed curve it means not deformable to a point or a puncture). For a hyperbolic $S$, let $\mathcal{C}(S, \partial S)$ denote the set of homotopy classes of curve systems under homotopies that keep the endpoints (if any) on the boundary. (We admit non-simple curves to the homotopy classes for convenience.) Let $\mathcal{C}(S) \subset \mathcal{C}(S, \partial S)$ denote the subset of curve systems represented by unions of simple closed curves. Note that $\mathcal{C}(S)$ includes curves that are homotopic to components of $\partial S$. These are called peripheral, and to exclude them we define $\mathcal{C}_{0}(S)$ to be the subset of $\mathcal{C}(S)$ consisting of curve systems with no peripheral components. Also define (for brief use in $\S 3) \mathcal{C}^{\prime}(S, \partial S)$ to be the set of homotopy classes of curve systems which are allowed to terminate in punctures as well as boundary components.

For an annulus $A$, we make a different definition: Let $\mathcal{C}(A, \partial A)$ denote the set of curve systems on $A$ with equivalence under homotopies that fix the endpoints.

We call a subsurface $M \subset S$ hyperbolic if no component of $\partial M$ is homotopically trivial or homotopic to another component of $\partial M$. Equivalently, if $\sigma$ denotes any smooth non-positively curved metric on $S$ in which the boundary is geodesic (or convex), then $M$ is isotopic to a subsurface $M^{\sigma}$ whose boundary is geodesic in $\sigma$.

Given a hyperbolic subsurface $M$, there is a natural map

$$
\mathcal{R}=\mathcal{R}_{M}: \mathcal{C}(S, \partial S) \rightarrow \mathcal{C}(M, \partial M),
$$

defined as follows: Let $\sigma$ be a standard hyperbolic metric on $S$. If $\gamma$ is a curve system on $S$ let $\gamma^{\sigma}$ denote its representative of shortest $\sigma$-length (see $\S 2.3$ ). Let $\mathcal{R}([\gamma])$ be the class represented by $\gamma^{\sigma} \cap M^{\sigma}$. It is not hard to see that $\mathcal{R}$ does not depend on the choice of $\sigma$.

$\S 2.3$. Lengths and geodesic representatives. For any metric $\sigma$ on $S$ and any curve $\alpha$ we denote by $\ell_{\sigma}(\alpha)$ the length of $\alpha$ as measured in $\sigma$. If $[\gamma] \in \mathcal{C}(S, \partial S)$ then $\gamma^{\sigma}$ denotes a representative of minimal length - if $\sigma$ is a standard hyperbolic metric then this is unique, and orthogonal to $\partial S$. For an annulus $A$ where the homotopies 
must fix endpoints, there is also a minimal representative $\gamma^{\sigma}$, but it may not be orthogonal to $\partial S$. In both cases we may denote $\ell_{\sigma}\left(\gamma^{\sigma}\right)$ by $\ell_{\sigma}([\gamma])$. Note also that any $\gamma \in \mathcal{C}^{\prime}(S, \partial S)$ has a unique geodesic representative $\gamma^{\sigma}$, though its length may be infinite.

Extremal length. For a metric $\sigma$ and any class $[\gamma]$ of curves in $S$, we can define the extremal length $\lambda_{\sigma}([\gamma])$ as

$$
\lambda_{\sigma}([\gamma])=\lambda_{S, \sigma}([\gamma])=\sup _{\sigma^{\prime} \in[\sigma]} \frac{\inf _{\gamma^{\prime} \in[\gamma]} \ell_{\sigma^{\prime}}^{2}\left(\gamma^{\prime}\right)}{\operatorname{Area}\left(\sigma^{\prime}\right)} .
$$

Where the supremum is taken over all metrics $\sigma^{\prime}$ conformally equivalent to $\sigma$. (See Ahlfors [2].) In particular, $\lambda_{\sigma}$ only depends on the conformal class of $\sigma$.

If $A$ is an annulus with conformal structure $[\sigma]$ then its conformal modulus $m(A, \sigma)$ (or $m(A)$ if $\sigma$ is implicit) is the extremal length of the class of arcs joining its boundaries. The extremal length of the class of simple closed curves homotopic to either boundary is $1 / m(A)$. A standard Euclidean metric on $A$ is unique in its conformal class up to constant multiple, and realizes the supremum in the definition of the modulus. Thus $m(A)=H / L$ where $H$ is the distance between the boundaries in this metric, and $L$ is the length of either boundary.

$\S 2.4$. The Thick-Thin decomposition. Fixing a standard hyperbolic metric $\sigma$ on $S$, the $\epsilon$-thin part of $(S, \sigma)$ is the subset of $S$ where the injectivity radius is at most $\epsilon$. The closure of its complement is called the $\epsilon$-thick part. If $\epsilon$ is less than the Margulis constant for $\mathbf{H}^{2}$ (see e.g. [24]), then the $\epsilon$-thin part consists of compact annular neighborhoods of short geodesics, and non-compact cuspidal components, or neighborhoods of punctures. Let us call a compact annulus $A$ of the thin part peripheral if its core is homotopic to $\partial S$ (in which case one of its boundary components lies on $\partial S$ ), and internal otherwise. In either case call a boundary component of $A$ internal if it lies in the interior of $S$.

For our later convenience we shall describe the following slight variation on the standard decomposition, which we shall call the $\left(\epsilon_{0}, \epsilon_{1}\right)$ collar decomposition, for two numbers $0<\epsilon_{1}<\epsilon_{0}$ less than the Margulis constant. Let $A_{1}, \ldots, A_{k}$ denote the annular neighborhoods of the geodesics of length $\delta_{i} \leq \epsilon_{1}$, such that the internal boundary components of $A_{i}$ have length $\epsilon_{0}$. It is not hard to see that the $A_{i}$ are contained in separate components of the $\epsilon_{0}$-thin part, and that $S-\cup A_{i}$, minus neighborhoods of the cusps, are in the $\epsilon_{1}^{\prime}$-thick part, for some $\epsilon_{1}^{\prime}$ possibly slightly smaller than $\epsilon_{1}$.

Let $\mathcal{A}$ denote the set of $A_{i}$, and let $\mathcal{P}$ denote the set of closures $P$ of components of $S-\cup A_{i}$. We call $\mathcal{P}$ the set of hyperbolic components of the decomposition. Let $\mathcal{Q}=\mathcal{P} \cup \mathcal{A}$ denote the entire decomposition.

It is well-known that $\epsilon_{0}$ may be chosen sufficiently small (independently of the surface) that, for any simple geodesic $\gamma$ in $S$, the components of $\gamma \cap A_{j}$ are either segments that traverse $A_{j}$ from one boundary to the other, or are equal to the geodesic core of $A_{j}$. 
One can see from an explicit computation (in the upper half-plane, for example) that the modulus of each $A_{i}$ is

$$
m\left(A_{i}\right)=\frac{\pi}{\delta_{i}}-\frac{2}{\epsilon_{0}}
$$

and we can guarantee by choosing $\epsilon_{1}$ sufficiently small that $m\left(A_{i}\right)$ is larger than any given constant $m_{0}$.

$\S 2.5$. The Teichmüller metric, and Kerckhoff's theorem. The Teichmüller distance on $\mathcal{T}(S)$ is defined for two conformal structures $[\sigma],[\tau]$ as

$$
d_{\mathcal{T}(S)}(\sigma, \tau)=\frac{1}{2} \log K(\sigma, \tau)
$$

where $K \geq 1$ is the least number such that there exists a homeomorphism homotopic to the identity on $S$ which is a $K$-quasiconformal map between the $\sigma$ and the $\tau$ conformal structures. We shall be using a different characterization of $K(\sigma, \tau)$, due to Kerckhoff, namely:

Theorem 2.1. For a surface of finite topological type $S$, the following holds:

$$
K(\sigma, \tau)=\sup _{\alpha \in \mathcal{C}(S, \partial S)} \frac{\lambda_{S, \sigma}(\alpha)}{\lambda_{S, \tau}(\alpha)}
$$

Remarks. Kerckhoff proved this in [12] for a closed surface $S$. However the same techniques apply to surfaces of finite analytic type, and the case with boundary can also be deduced easily using a doubling argument (see e.g. Abikoff [1, §II.1.5] for an example of such arguments). An interesting feature of the equivalence of the two definitions of $K$ is that the first one is fairly easily seen to be symmetric in $\sigma$ and $\tau$ (since the inverse of a $K$-quasiconformal map is also $K$-quasiconformal), but the symmetry of the second is less evident. We shall use this to advantage in section 6 .

The Teichmüller space of the torus is isometric to the hyperbolic plane $\mathbf{H}^{2}$, and Kerckhoff's theorem in this case can be written as a simple formula, after proper interpretation. We will use this formula not for the torus but in conjunction with twisting numbers and the annulus contributions to extremal length, $\lambda_{A, \sigma}$ (definition (4.3)) in section 6. For the sake of exposition we shall give an elementary proof:

Lemma 2.2. Let $\mathbf{H}$ denote the upper half-plane $\{z: \operatorname{Im}(z)>0\}$, and define for $z_{j}=x_{j}+i y_{j}$

$$
K\left(z_{1}, z_{2}\right)=\sup _{t \in \mathbf{R}} \frac{y_{2}+\left(t+x_{2}\right)^{2} / y_{2}}{y_{1}+\left(t+x_{1}\right)^{2} / y_{1}}
$$

Then

$$
d_{\mathbf{H}}\left(z_{1}, z_{2}\right)=\frac{1}{2} \log K\left(z_{1}, z_{2}\right)
$$

where $d_{\mathbf{H}}\left(z_{1}, z_{2}\right)$ is the hyperbolic distance function.

(Note: actually the common definition of $d_{\mathbf{H}}$ does not include the factor of a half; however we will retain it in order to be consistent with (2.3).) 
Proof of 2.2: Consider first the case that $z_{1}$ and $z_{2}$ are purely imaginary. It is an easy exercise that $K\left(i y_{1}, i y_{2}\right)=\max \left\{y_{2} / y_{1}, y_{1} / y_{2}\right\}$. Since $\frac{1}{2}\left|\log \left(y_{2} / y_{1}\right)\right|$ is exactly the hyperbolic distance function, this completes the proof in this case.

Since for general $z_{1}, z_{2} \in \mathbf{H}$ there is always some hyperbolic isometry $A \in \mathrm{SL}_{2}(\mathbf{R})$ such that $A\left(z_{1}\right), A\left(z_{2}\right)$ are purely imaginary, it would suffice to show that $K$ is invariant under the action of $\mathrm{SL}_{2}(\mathbf{R})$.

One can see this conveniently by reducing it to a linear algebra problem. Let $\widehat{\mathbf{H}}$ denote $\left\{(\alpha, \beta) \in \mathbf{C}^{2}: \operatorname{Im}(\beta / \alpha)>0\right\}$. For $(\alpha, \beta) \in \widehat{\mathbf{H}}$ and $u, v \in \mathbf{R}$, define

$$
\lambda_{\alpha, \beta}(u, v)=\frac{|u \alpha+v \beta|^{2}}{\operatorname{Im}(\beta \bar{\alpha})} .
$$

Then define $\widehat{K}$ on $\widehat{\mathbf{H}} \times \widehat{\mathbf{H}}$ by

$$
\widehat{K}\left(\alpha_{1}, \beta_{1}, \alpha_{2}, \beta_{2}\right)=\sup _{u, v \in \mathbf{R}} \frac{\lambda_{\alpha_{2}, \beta_{2}}(u, v)}{\lambda_{\alpha_{1}, \beta_{1}}(u, v)} .
$$

One can check that the left action of $\mathrm{SL}_{2}(\mathbf{R})$ on $\mathbf{C}^{2}$ (viewed as column vectors) preserves $\operatorname{Im}(\beta \bar{\alpha})=|\alpha|^{2} \operatorname{Im}(\beta / \alpha)$, and therefore in particular preserves $\widehat{\mathbf{H}}$. Furthermore note that the denominator of $\lambda_{\alpha, \beta}(u, v)$ is the squared norm of an inner product $(u, v) \cdot(\alpha, \beta)$, so it is easy to see that $\lambda_{A(\alpha, \beta)}(u, v)=\lambda_{\alpha, \beta}((u, v) A)$. It follows that $A \in \mathrm{SL}_{2}(\mathbf{R})$ leaves $\widehat{K}$ invariant. since the supremal ratio over all $(u, v)$ is the same as the supremum over all $(u, v) A$.

It is an easy computation to show that $\lambda_{1, z}(t, 1)=y+(t+x)^{2} / y$ if $z=x+i y$, and that $\lambda_{1, \beta / \alpha}(u, v)=\lambda_{\alpha, \beta}(u, v)$. Moreover, $\lambda_{\alpha, \beta}(c u, c v)=c^{2} \lambda_{\alpha, \beta}(u, v)$. It follows that

$$
\widehat{K}\left(\alpha_{1}, \beta_{1}, \alpha_{2}, \beta_{2}\right)=K\left(\beta_{1} / \alpha_{1}, \beta_{2} / \alpha_{2}\right) .
$$

The map $(\alpha, \beta) \mapsto \beta / \alpha$ is a fibration of $\widehat{\mathbf{H}}$ over $\mathbf{H}$, which takes the linear action of $\mathrm{SL}_{2}(\mathbf{R})$ upstairs to its action by hyperbolic isometries downstairs. We conclude that $K$ is also invariant under $\mathrm{SL}_{2}(\mathbf{R})$.

Remark. One can give a geometric interpretation to this proof by associating to $(\alpha, \beta) \in \widehat{\mathbf{H}}$ a torus, obtained by identifying opposite sides on a parallelogram spanned by $\alpha$ and $\beta$ in $\mathbf{C}$. The quantity $\operatorname{Im}(\beta \bar{\alpha})$ is the area of this parallelogram. For $u, v \in \mathbf{Z}$, the quantity $\lambda_{\alpha, \beta}(u, v)$ is the extremal length of the homotopy class in the torus represented by $(u, v)$ in the generators $\alpha$ and $\beta$ (for general $u, v \in \mathbf{R}$, one can give an interpretation in terms of measured foliations on the torus). Now $\widehat{K}$ can be seen as a supremum of extremal length ratios, just as in Kerckhoff's theorem. The fibration from $\widehat{\mathbf{H}}$ to $\mathbf{H}$ is just identification under conformal equivalence. 


\section{$\S 3$. Twisting numbers and Fenchel-Nielsen coordinates}

In this section we will describe a measure, called $t_{\gamma, \sigma}(\alpha)$, for the amount that a simple curve $\alpha$ in a hyperbolic surface $(S, \sigma)$ "twists" around another curve $\gamma$. In particular if $\gamma$ is short then this number will approximate the amount of twisting that $\alpha$ undergoes in the thin annulus corresponding to $\gamma$, and this will determine the contribution of this annulus to the extremal length of $\alpha$. At the end of the section we will relate this construction to the familiar Fenchel-Nielsen twist coordinates.

Let $\sigma$ be a standard hyperbolic metric on $S$. Let $\gamma$ denote a (homotopically non-trivial) oriented simple closed curve, and let $\alpha$ denote any element of $\mathcal{C}^{\prime}(S, \partial S)$, which intersects $\gamma$. Let $x$ be a point of the intersection $\gamma^{\sigma} \cap \alpha^{\sigma}$. In the universal cover $\widetilde{S}$ of $S$, which is a convex subset of $\mathbf{H}^{2}$, let $L_{\gamma}$ be a geodesic in the lift of $\gamma^{\sigma}$, and $L_{\alpha}$ be a geodesic in the lift of $\alpha^{\sigma}$ (extended to an infinite geodesic if it meets $\partial \widetilde{S}$ ), such that $L_{\gamma}$ and $L_{\alpha}$ meet in a point that projects to $x$. Identify $L_{\gamma}$ isometrically with $\mathbf{R}$, consistent with the orientation of $\gamma$, and let let $a_{R}, a_{L}$ be the endpoints of $L_{\alpha}$ to the right and left, respectively, of $L_{\gamma}$. Let $p_{\gamma}: \mathbf{H}^{2} \cup S^{1} \rightarrow L_{\gamma}$ be the orthogonal projection, and define the (signed) twisting number

$$
t_{\gamma, \sigma}^{\prime}(\alpha, x)=\frac{p_{\gamma}\left(a_{R}\right)-p_{\gamma}\left(a_{L}\right)}{\ell_{\sigma}(\gamma)}
$$

(see figure 1). It is clear that the definition does not depend on the particular choice of lifts.

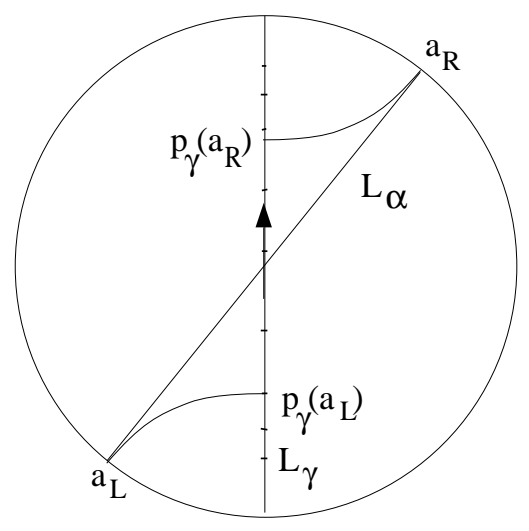

Figure 1

Lemma 3.1. Let $\gamma$ be a homotopically non-trivial oriented simple closed curve, $\alpha \in \mathcal{C}(S, \partial S)$, and $\sigma$ a standard hyperbolic metric on $S$. If $x$ and $x^{\prime}$ are two points of $\gamma^{\sigma} \cap \alpha^{\sigma}$, then

$$
\left|t_{\gamma, \sigma}^{\prime}(\alpha, x)-t_{\gamma, \sigma}^{\prime}\left(\alpha, x^{\prime}\right)\right| \leq 1
$$

Proof of 3.1: Let $g$ be the indivisible element of $\pi_{1}(S)$ whose axis is $L_{\gamma}$. Choosing appropriate lifts $L_{\alpha}$ and $L_{\alpha}^{\prime}$ corresponding to $x$ and $x^{\prime}$, we may assume that $L_{\alpha}^{\prime}$ is trapped between $L_{\alpha}$ and $g\left(L_{\alpha}\right)$. The proof is completed by figure 2 . 


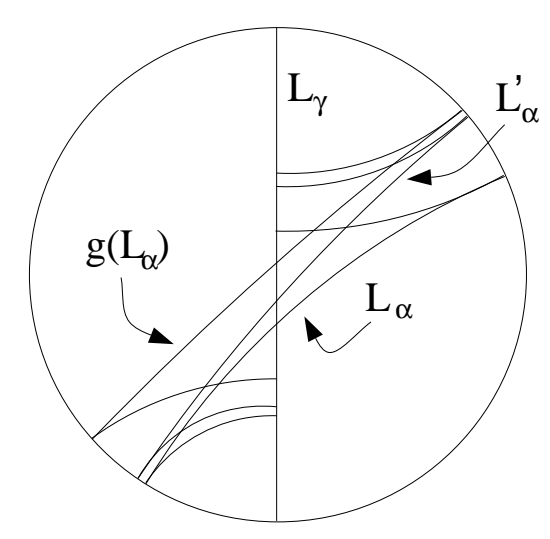

FiguRe 2

Thus it is useful to define the quantity

$$
t_{\gamma, \sigma}(\alpha)=\min _{x} t_{\gamma, \sigma}^{\prime}(\alpha, x)
$$

where the infimum is taken over all $x$ in $\gamma^{\sigma} \cap \alpha^{\sigma}$.

We can also measure twisting inside an annulus. If $A$ is an annulus with conformal structure $[\sigma]$ then let $\sigma$ be a standard Euclidean metric on $A$ in this conformal class. The univeral cover of $A$ is isometric to a strip $[0, L] \times \mathbf{R}$ in the plane, with fundamental domain of height $H$. If $\alpha$ is a curve connecting the boundaries of $A$, it lifts to a curve with endpoints $\left(0, y_{0}\right)$ and $\left(L, y_{1}\right)$, and we may define

$$
t_{A, \sigma}(\alpha)=\left(y_{1}-y_{0}\right) / H
$$

(the sign of $t$ is again determined after a choice of orientation of $A$ ).

Now consider again a standard hyperbolic metric $\sigma$ on $S$, and an annulus $A$ of the $\left(\epsilon_{0}, \epsilon_{1}\right)$ collar decomposition of $(S, \sigma)$. If $\alpha \in \mathcal{C}(S)$ then its geodesic representative $\alpha^{\sigma}$ intersects $A$ in several components, whose twists differ by no more than one. Thus we may take $t_{A, \sigma}(\alpha)$ to be the minimum of these. Note in particular that if $A$ is peripheral then $t_{A, \sigma}(\alpha)=0$ since $\alpha^{\sigma}$ is perpendicular to $\partial A$.

Lemma 3.2. There exist $\epsilon_{0}, \epsilon_{1}$ less than the Margulis constant such that, for any standard hyperbolic metric $\sigma$ on a surface $S$, if $A$ is an $\left(\epsilon_{0}, \epsilon_{1}\right)$ collar with (nonperipheral) core $\gamma$ and $\alpha$ is any element of $\mathcal{C}(S)$ that crosses $\gamma$,

$$
\left|t_{A, \sigma}(\alpha)-t_{\gamma, \sigma}(\alpha)\right| \leq 1
$$

Proof of 3.2: In case $A$ is peripheral both twists are 0, so let us assume $A$ is internal. Consider figure 3 , where in the upper half-plane model of $\mathbf{H}^{2}$ we place a lift $L_{\gamma}$ of $\gamma^{\sigma}$, and the corresponding lift $\widetilde{A}$ of $A$. Pick $x \in \gamma^{\sigma} \cap \alpha^{\sigma}$ and choose a component $L_{\alpha}$ of the lift of $\alpha^{\sigma}$ which intersects $L_{\gamma}$ in a point projecting to $x$. Let $\alpha_{x}$ denote 
the component of $\alpha^{\sigma} \cap A$ which contains $x$. We may arrange the figure so that $L_{\alpha}$ exits $\widetilde{A}$ as shown, terminating at infinity in the interval $J$.

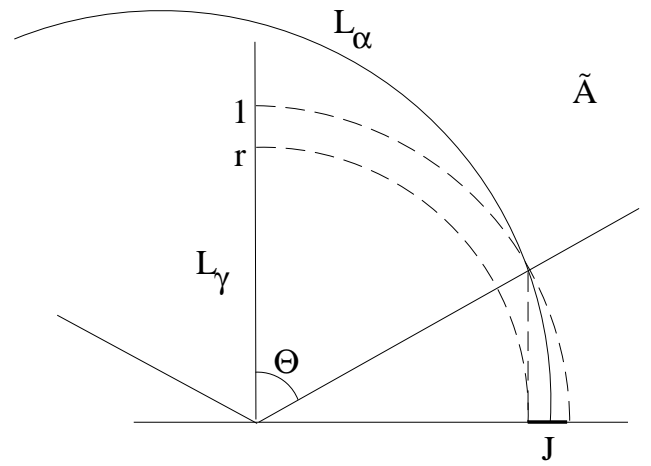

FIGURE 3

The angle $\theta$ between $L_{\gamma}$ and $\partial \widetilde{A}$ satisfies $\cos \theta \leq \epsilon_{1} / \epsilon_{0}$, and the position $r$ of the projection of the endpoint of $L_{\alpha}$ satisfies $1 \geq r>\sin \theta$. It follows immediately that

$$
0 \leq t_{\gamma, \sigma}^{\prime}(\alpha, x)-t_{A, \sigma}\left(\alpha_{x}\right)<\frac{1}{\epsilon_{1}}\left|\log \left(1-\epsilon_{1}^{2} / \epsilon_{0}^{2}\right)\right| .
$$

Thus given $\epsilon_{0}$ we may choose $\epsilon_{1}$ sufficiently small that this is at most 1 . Since both $t_{\gamma, \sigma}^{\prime}(\alpha, x)$ and $t_{A, \sigma}\left(\alpha_{x}\right)$ are minimized for the same $x$ (both depend monotonically on the angle at which $\gamma^{\sigma}$ and $\alpha_{x}$ cross), we have the desired inequality.

The following lemma observes that for a thin annulus $A$ any twisting number can be achieved, up to an additive constant, by some curve that spends "most" of its time in $A$. This fact will be used in the proof of theorem 6.1 .

Lemma 3.3. Let $(Q, \tau)$ be a surface of finite topological type with standard hyperbolic metric $\tau$. Let $\epsilon_{0}>\epsilon_{1}>0$ be as in lemma 3.2, and fix $\ell_{1}>0$. Suppose that $\ell_{\tau}(\gamma) \leq \ell_{1}$ for all boundary components $\gamma$ of $Q$, and let $A \subset Q$ be an internal annulus of the $\left(\epsilon_{0}, \epsilon_{1}\right)$ collar decomposition. Given $t \in \mathbf{R}$ there exists $\alpha \in \mathcal{C}(Q)$ such that

$$
\left|t_{A, \tau}(\alpha)-t\right| \leq c_{1}
$$

and $\alpha$ consists of one or two arcs traversing A, together with one or two segments of hyperbolic length at most $c_{2}$. The constants $c_{1}, c_{2}$ depend only on $\epsilon_{0}, \epsilon_{1}, \ell_{1}$ and $\chi(Q)$.

Proof of 3.3: We claim that, for some $r_{1}$ depending on $\epsilon_{0}, \epsilon_{1}, \ell_{1}$ and $\chi(Q)$, there is, for each boundary component of $A$, a geodesic arc outside $A$ of length at most $r_{1}$, which either joins that component to the other, or to itself. To see this, let $A_{r}$ denote the $r$-neighborhood of $A$ in $Q ; A_{r}$ is the union of $A$ with two pieces, each adjacent to a boundary component. If at least one of them is a collar, i.e. an 
embedded annulus of radius $r$ in the interior of $Q$, we have $\operatorname{Area}\left(A_{r}\right)>\epsilon_{1} e^{r}$, and since $\operatorname{Area}(Q) \leq 2 \pi|\chi(Q)|$ we have an upper bound $r<r_{0}=\log 2 \pi|\chi(Q)| / \epsilon_{1}$. Thus, if $r \geq r_{0}$ then both added pieces fail to be collars. If for some $r \leq r_{0}$ a collar meets itself or the other collar we have the desired geodesic arc of length $r_{1}=2 r_{0}$. The other possibility is for a collar to hit the boundary at some $r \leq r_{0}$. In this case there is an arc of length at most $r_{1}=2 r_{0}+\ell_{1}$ which runs once around the boundary and back to $A$.

Now if there is an arc joining both components, we can take $\alpha_{0}$ to be the union of this short arc with an arc that runs through $A$. If there are two arcs, joining each boundary to itself, we can join these arcs to a union of two parallel arcs through $A$. Applying $n$ Dehn twists around the core of $A$ to $\alpha_{0}$ will clearly increase (or decrease) its twisting number by about $n$ (see also lemma 3.5 ), so that by an approriate twist we may obtain $\alpha$ with the desired twisting number.

In the proof of corollary 5.4, we will need to measure twisting using a hyperbolic metric that is not quite standard. In particular, suppose that $\sigma$ is a standard hyperbolic metric on $S$ and that $P$ is a subsurface consisting of a union of components of the $\left(\epsilon_{0}, \epsilon_{1}\right)$ collar decomposition, such that any annular components in $P$ are non-peripheral in $P$. The metric $\sigma$ restricted to $P$ is not standard, but it is not too far from it. Let $\sigma^{\prime}$ be the standard hyperbolic metric on $P$ which is conformally equivalent to $\sigma$. Now if $\gamma \in P$ is a non-peripheral geodesic of length $\ell_{\sigma}(\gamma)<\epsilon_{1}$, we can measure twisting around $\gamma$ with respect to both metrics. The following lemma compares the two:

Lemma 3.4. Let $P, S, \sigma$ and $\sigma^{\prime}$ be as above. There exist $\epsilon_{0}, \epsilon_{1}$ sufficiently small and $c>0$ such that, if $\ell_{\sigma}\left(\gamma^{\sigma}\right)<\epsilon_{1}$ for a non-peripheral simple closed curve $\gamma \subset P$, then

$$
\left|t_{\gamma, \sigma}(\beta)-t_{\gamma, \sigma^{\prime}}\left(\mathcal{R}_{P}(\beta)\right)\right| \leq c
$$

for any $\beta \in \mathcal{C}(S, \partial S)$.

Proof of 3.4: We shall give the proof in the case that $S$ has no boundary. The general case can be reduced to this by means of a doubling argument. Let $\beta^{\sigma}$ be the $\sigma$ geodesic representative of $\beta$. Let $P_{0}$ be the complement in $P$ of $\epsilon_{\mathcal{M}}$-thin annulus neighborhoods of $\partial P$ (measured in $\hat{\sigma}$ ), where $\epsilon_{\mathcal{M}}>\epsilon_{0}$ is the Margulis constant. We claim that, given $\nu>0$ there is a choice of $\epsilon_{0}$ so that in $P_{0}$ the ratio $\sigma / \hat{\sigma}$ is $\nu$-close to 1 , and its derivatives (in normal $\hat{\sigma}$ coordinates) are bounded by $\nu$. This will imply that $\beta^{\sigma} \cap P_{0}$ is a curve system with very low $(O(\nu))$ curvature in the metric $\hat{\sigma}$. Thus the $\hat{\sigma}$-geodesic $\hat{\beta}_{0}$ homotopic to $\beta^{\sigma} \cap P_{0}$ rel endpoints is $O(\nu)$-close to it. Now, we are interested in $\mathcal{R}_{P}(\beta)^{\hat{\sigma}}$, the $\hat{\sigma}$-geodesic representative of $\beta^{\sigma} \cap P$, rel $\partial P$. But by lemma 4.5 applied in $(P, \hat{\sigma})$, the intersection $\mathcal{R}_{P}(\beta)^{\hat{\sigma}} \cap P_{0}$ is obtained by a bounded homotopy from $\hat{\beta}_{0}$. It follows that the twisting number around $\gamma$, which is certainly in $P_{0}$, has not changed by more than a bounded amount. 
It remains to prove our claim about $\sigma / \hat{\sigma}$, but this is an elementary argument in complex analysis. If we consider the universal coverings of $P$ in (subsets of) the unit disk which are obtained from the two metrics $\sigma$ and $\hat{\sigma}$, we see that the identity on $P$ lifts to a conformal map $\Phi$ between these covers. For $x \in P_{0}$, the hyperbolic distance to $\partial P$, in either metric, is roughly $\log \epsilon_{\mathcal{M}} / \epsilon_{0}$, which we can make as large as we please. If $x$ lifts to 0 in both covers we see (using the formulas for hyperbolic distance) that $\Phi$ must map a disk of radius roughly $\left(\epsilon_{\mathcal{M}}-\epsilon_{0}\right) /\left(\epsilon_{\mathcal{M}}+\epsilon_{0}\right)$ univalently over a disk of similar radius. The Schwarz lemma applied to $\Phi$ and $\Phi^{-1}$ implies that $\left|\Phi^{\prime}(z)\right|$ is close to 1 in a neighborhood of 0 , and an application of the Cauchy integral formula serves to bound $\Phi^{\prime \prime}(0)$. Our claim about $\sigma / \hat{\sigma}$ follows.

Recall now the construction of Fenchel-Nielsen coordinates on a Teichmüller space $\mathcal{T}(S)$ (see e.g. [1]) Let $\gamma_{1}, \ldots, \gamma_{n}$ denote a system of oriented simple closed curves which cuts $S$ into a union of pairs of pants. Suppose, to start, that $S$ has no punctures and $p$ boundary components, so that $n=-3 \chi(S) / 2$ and each pair of pants is compact. For any standard hyperbolic structure $\sigma$ on $S$ we immediately obtain $n$ positive numbers $\left\{\ell_{\sigma}\left(\gamma_{i}^{\sigma}\right)\right\}$, the length parameters. Another $n-p$ real numbers describe "twisting" parameters for the gluings of the pairs of pants. Let us make this concrete as follows.

A pair of pants $P$ with boundary curves $\gamma_{1}, \gamma_{2}, \gamma_{3}$ contains three unique homotopy classes of simple properly embedded $\operatorname{arcs} \alpha_{12}, \alpha_{23}, \alpha_{13}$ (the "seams"), such that $\alpha_{i j}$ joins $\gamma_{i}$ to $\gamma_{j}$. Fix a set of representatives of the seams which match on opposite sides of each non-peripheral $\gamma_{i}$. This determines a system of curves $\mu$ in $\mathcal{C}(S, \partial S)$. We call the (oriented) $\gamma_{i}$ together with $\mu$ a marking, and note that it is purely topological information.

Now for any standard hyperbolic structure $\sigma$ on $S$, the seams have geodesic representatives $\alpha_{i j}^{\sigma}$ which are orthonal to $\gamma_{i}^{\sigma}$ and $\gamma_{j}^{\sigma}$. The seams cut each pair of pants into two congruent right-angled hexagons, and in particular they bisect the boundary components. Now for each $\gamma_{j}$ and each pair of corresponding seam endpoints there is a unique geodesic path along $\gamma_{j}$ that must be spliced between the endpoints, so that the resulting curve is in the homotopy class $\mu$. The length $m_{j}(\sigma)$ of this path (which can be given a sign, since $\gamma_{j}$ is oriented) is the same for both pairs of seam endpoints on $\gamma_{j}$. We define our twist parameter $s_{j}(\sigma)$ to be $m_{j}(\sigma) / \ell_{\sigma}\left(\gamma_{j}\right)$.

This construction gives a homeomorphism $F: \mathcal{T}(S) \rightarrow \mathbf{R}_{+}^{n} \times \mathbf{R}^{n-p}$, taking $\sigma$ to $\left(\ell_{\sigma}\left(\gamma_{1}\right), \ldots, \ell_{\sigma}\left(\gamma_{n}\right), s_{1}(\sigma), \ldots, s_{n-p}(\sigma)\right)$. We note also that a positive Dehn twist on $\gamma_{j}$ has the effect of incrementing $s_{j}(\sigma)$ by one and leaving the other coordinates invariant.

In the case with punctures the construction is similar, except that one or two ends of a pair of pants may be a puncture, and we allow the seams to be non-compact arcs terminating in boundaries or punctures, so that $\mu$ is in $\mathcal{C}^{\prime}(S, \partial S)$.

To conclude this section, we give a comparison between our twisting numbers 
and the Fenchel-Nielsen twist coordinates.

Lemma 3.5. Let $\gamma_{1}, \ldots, \gamma_{n}$ and $\mu$ be a marking for $S$, and $s_{j}: \mathcal{T}(S) \rightarrow \mathbf{R}$ be the associated twist coordinates. Then for any standard hyperbolic metric $\sigma$ on $S$,

$$
\left|s_{j}(\sigma)-t_{\gamma_{j}, \sigma}(\mu)\right| \leq 1 \text {. }
$$

Furthermore, given any $\alpha \in \mathcal{C}(S, \partial S)$ and two standard hyperbolic metrics $\sigma$ and $\sigma^{\prime}$,

$$
\left|\left(t_{\gamma_{j}, \sigma}(\alpha)-t_{\gamma_{j}, \sigma^{\prime}}(\alpha)\right)-\left(s_{j}(\sigma)-s_{j}\left(\sigma^{\prime}\right)\right)\right| \leq 4
$$

Proof of 3.5: To obtain the first inequality, lift $\gamma_{j}^{\sigma}$ to a geodesic $L_{j}$ in $\mathbf{H}^{2}$, and consider a lift $\widetilde{\mu}$ of a component of $\mu^{\sigma}$ that intersects $L_{j}$. This lift is homotopic to a curve that follows two (lifts of) seam curves $\alpha_{j k}^{\sigma}, \alpha_{j m}^{\sigma}$ whose endpoints on $L_{j}$ are separated by $s_{j}(\sigma)$. The other endpoints of these seams meet lifts $L_{k}$ and $L_{m}$ of $\gamma_{k}^{\sigma}, \gamma_{m}^{\sigma}$. Sinc $L_{k}$ (resp. $L_{m}$ ) is disjoint from its translates, its projection to $L_{j}$ has length at most $\ell_{\sigma}\left(\gamma_{j}\right)$. The endpoints of $\widetilde{\mu}$ at infinity must be separated from $L_{j}$ by $L_{k}$ and $L_{m}$, respectively. It follows that the length of the projection of $\mu$ to $L_{j}$, divided by $\ell_{\sigma}\left(\gamma_{j}\right)$, differs from $s_{j}(\sigma)$ by at most 1 .

For the rest of the lemma we note the fact that, for any two curve systems $\alpha, \beta \in \mathcal{C}^{\prime}(S, \partial S)$, the difference $t_{\gamma_{j}, \sigma}(\alpha)-t_{\gamma_{j}, \sigma}(\beta)$ is independent of $\sigma$ up to a bounded error (of 1 ). This is because we can detect this difference topologically: lifting to the universal cover, let $L_{\alpha}$ be a lift of a component of $\alpha$ which crosses a lift $L_{j}$ of $\gamma_{j}$. The translates of $L_{\alpha}$ along $L_{j}$ divide the plane into a sequence of consecutive strips, and a component of a lift of $\beta$ that crosses $L_{j}$ meets some number $\delta$ of these strips. (The number obtained from a different lift of $\beta$ may differ by at most 1 ). It is easy to see that $\delta$, appropriately signed, gives this twist difference, up to an error of 1 .

The second inequality now follows from this fact applied to $\alpha$ and $\mu$, together with the first inequality.

\section{$\S 4$. Basic extremal length estimates}

In this section we shall discuss and derive some fairly well-known estimates for extremal lengths, which will be the building blocks of the proof in section 5 .

Here and in the rest of the paper, we shall use " $a \asymp b$ " to mean that the ratio $a / b$ is bounded above and below by positive constants, which usually depend on topological data, and on previously chosen constants. Similarly, " $a \prec b$ " and " $b \succ a$ " mean that $a / b$ is bounded above. Where necessary we will indicate explicitly the dependence of the constants. 
$\S 4.1$. Spacing and upper bounds. Lower bounds on extremal lengths are relatively easy to obtain, by exhibiting some metric and computing the length in that metric. The following lemma is helpful for obtaining upper bounds.

For a representative curve $\hat{\beta}$ of a class $\beta$ in $C(S, \partial S)$ and a metric $\sigma$ on $S$, define the spacing $\nu_{S, \sigma}(\hat{\beta})$ as the supremum of numbers $\nu$ such that the $\nu$-neighborhood $\mathcal{N}_{\nu}(\hat{\beta})$ is a standard product $\hat{\beta} \times[0,1]$. We have:

Lemma 4.1. Given a metric $\sigma$ on $S$ and a representative $\hat{\beta}$ of $\beta \in C(S, \partial S)$, the extremal length of $\beta$ is bounded by

$$
\lambda_{S, \sigma}(\beta) \leq \frac{\operatorname{Area}(S, \sigma)}{4 \nu_{S, \sigma}^{2}(\hat{\beta})} .
$$

Proof of 4.1: Let $\beta_{1}, \ldots, \beta_{n}$ denote the components of $\hat{\beta}$. The neighborhoods $R_{i}=$ $\mathcal{N}_{\nu}\left(\beta_{i}\right)$ for any $\nu<\nu_{S, \sigma}(\hat{\beta})$ are, by assumption, either annuli or rectangles. In either case, the extremal length of the family of transverse arcs $\alpha_{i}$ connecting the boundaries $\beta_{i} \times\{0,1\}$ is, applying the definition,

$$
\lambda_{R_{i}, \sigma}\left(\alpha_{i}\right) \geq \frac{4 \nu^{2}}{\operatorname{Area}\left(R_{i}, \sigma\right)} .
$$

Let $\left[\beta_{i}\right]$ denote the family of curves in $R_{i}$ freely homotopic to $\beta_{i}$ when $\beta_{i}$ is a closed curve, and homotopic with endpoints on the edges $\partial \beta_{i} \times[0,1]$ when $\beta_{i}$ is a segment. Then it is a standard fact that

$$
\lambda_{R_{i}, \sigma}\left(\left[\beta_{i}\right]\right)=1 / \lambda_{R_{i}, \sigma}\left(\alpha_{i}\right)
$$

This together with (4.1) bounds $\lambda_{R_{i}, \sigma}\left(\left[\beta_{i}\right]\right)$ from above. Now let $\beta_{R}$ denote the class of all curve systems that are a union of representatives from each $\left[\beta_{i}\right]$. Since the $R_{i}$ are all disjoint it is easy to see that

$$
\lambda_{\cup R_{i}, \sigma}\left(\beta_{R}\right)=\sum_{i=1}^{n} \lambda_{R_{i}, \sigma}\left(\left[\beta_{i}\right]\right)
$$

by an application of the Schwarz inequality (actually only the " $\leq$ " direction is needed, but the other direction is even easier).

The proof is completed by a standard monotonicity argument: Since the class $\beta$ in $\mathcal{C}(S, \partial S)$ is bigger than the class $\beta_{R}$ (it has representatives not limited to the $R_{i}$ ), its length in any metric is smaller. Furthermore the area of $S$ in any metric is at least that of $\cup R_{i}$. Thus $\lambda_{S, \sigma}(\beta) \leq \lambda_{\cup R_{i}, \sigma}\left(\beta_{R}\right)$, and the lemma follows. 
§4.2. Extremal length in hyperbolic subsurfaces. If a hyperbolic subsurface $M$ of $S$ is "insulated" from $S$ by sufficiently thick annuli on its boundary, then extremal lengths in $\mathcal{C}(S)$ and $\mathcal{C}_{0}(M)$ (curves that are non-peripheral in $M$ ) are approximately the same:

Lemma 4.2. [22, lemma 8.4] Let $(S, \sigma)$ be any Riemann surface. There exist $m_{0}$ and $C$, depending only on the topological type of $S$, such that if $M \subset S$ is a hyperbolic subsurface for which each component $\gamma$ of $\partial M$ bounds an annulus $A_{\gamma} \subset M$ with modulus $m\left(A_{\gamma}\right) \geq m_{0}$, then for any $\alpha \in \mathcal{C}_{0}(M)$,

$$
1 \leq \frac{\lambda_{M, \sigma}(\alpha)}{\lambda_{S, \sigma}(\alpha)} \leq C
$$

More generally, a curve in $S$ may cut through $M$, and if $M$ is a nicely chosen subsurface then we can say something about the extremal length of the intersection. For a standard hyperbolic metric $\sigma$, recall the $\left(\epsilon_{0}, \epsilon_{1}\right)$ collar decomposition $\mathcal{Q}=$ $\mathcal{P} \cup \mathcal{A}$. For a hyperbolic component $P \in \mathcal{P}$, define

$$
\lambda_{P, \sigma}(\beta)=\lambda_{P, \sigma}\left(\mathcal{R}_{P}(\beta)\right) .
$$

Note that, provided $\epsilon_{0}$ is chosen sufficiently small, $\mathcal{R}_{P}(\beta)$ is represented by $\beta^{\sigma} \cap P$. This hyperbolic geodesic also gives a good estimate for extremal length on $P$, via the following:

Lemma 4.3. Suppose that $\sigma$ is a standard hyperbolic metric for $S$, and suppose that $\ell_{\sigma}(\gamma) \leq \ell_{0}$ for any component $\gamma$ of $\partial S$. For any $\beta \in \mathcal{C}(S, \partial S)$ and a hyperbolic component $P$ of the $\left(\epsilon_{0}, \epsilon_{1}\right)$ collar decomposition,

$$
\lambda_{P, \sigma}(\beta) \asymp \ell_{\sigma}^{2}\left(\beta^{\sigma} \cap P\right)
$$

Where the multiplicative constants for $\asymp$ depend only on the topological type of $S$ and on $\epsilon_{0}, \epsilon_{1}$ and $\ell_{0}$.

Proof of 4.3: Because of the absence of thin parts in $P$ we can rearrange $\beta^{\sigma} \cap P$ to have sufficiently large spacing in the hyperbolic metric $\sigma$. Indeed,

Lemma 4.4. There is a curve system $\beta^{\prime}$ in $P$ homotopic to $\beta^{\sigma} \cap P$ by a homotopy that moves no point of $\beta^{\sigma} \cap \partial P$ more than halfway around the boundary, and such that

$$
\nu_{P, \sigma}\left(\beta^{\prime}\right) \geq \frac{c}{\ell_{\sigma}\left(\beta^{\sigma} \cap P\right)}
$$

where the constant $c$ depends only on the topological type of $S$, and on $\epsilon_{0}, \epsilon_{1}$ and $\ell_{0}$

Proof of 4.4: Subdivide $P$ into pairs of pants $Q_{1}, \ldots, Q_{p}$, using the shortest collection of pairwise disjoint simple geodesics in $P$. The number $p$ of pieces is given by $p=|\chi(P)|$, and we note that some pairs of pants may have one or two punctures rather than boundaries. The lengths of all boundary components of $Q_{i}$ are bounded below by $\epsilon_{1}$, and above by a constant $\ell_{1}$ depending on $\ell_{0}$ and the topological type of $S$. (This is a standard fact, whose proof is similar to the proof that the injectivity radius of $(S, \sigma)$ is bounded above in terms of the hyperbolic area of $S)$. 
Begin by performing a homotopy on $\beta^{\sigma} \cap Q_{j}$, for each $j$, which moves the points of $\beta^{\sigma} \cap \partial Q_{j}$ around $\partial Q_{j}$ until they are evenly spaced, and keeps the rest of the curve geodesic. Call the new curve $\beta_{1}$. Clearly this can be done so that no endpoint moves more than halfway around its boundary component. Furthermore, provided $\epsilon_{0}$ has been chosen sufficiently small, $\beta_{1}$ will still be contained in $P$.

The bound $\ell_{1}$ on boundary lengths means that the length of no arc has changed by more than an additive constant by this homotopy. It also means that there is a lower bound $\ell_{2}$ on the length of any path joining two boundaries of any $Q_{j}$. Furthermore no closed component of $\beta^{\sigma} \cap P$ has length less than $\epsilon_{1}$. We conclude that

$$
\ell_{\sigma}\left(\beta_{1}\right) \leq c_{1} \ell_{\sigma}\left(\beta^{\sigma} \cap P\right)
$$

where $c_{1}$ depends on the previous constants.

Now we deform $\beta_{1}$ in each $Q_{k}$ separately to obtain a curve $\beta_{2}$ with maximal spacing. Consider first the case where $\beta_{1} \cap Q_{k}$ has no closed curve components, and where $Q_{k}$ has three boundaries and no cusps.

One way to make the construction concrete is to form the following Voronoi diagram: For $i=1,2,3$ let $C_{i}$ be the collar defined by

$$
C_{i}=\left\{x \in Q_{k}: d\left(x, \gamma_{i}\right) \leq d\left(x, \gamma_{j}\right) \text { for } j \neq i\right\} .
$$

Then each $C_{i}$ is an annulus whose whose width at any point is bounded between two constants $c_{2}, c_{3}$, and whose boundary in the interior of $Q_{k}$ consists of two geodesic $\operatorname{arcs} \alpha_{i j}=C_{i} \cap C_{j}$ (for $j \neq i$ ) of length $s_{i j} \in\left[c_{4}, c_{5}\right]$ (The estimates here are uniform since the lengths of $\gamma_{i}$ are bounded above and below, so we have a compact set of possible pairs of pants. Alternatively one can obtain explicit estimates using hyperbolic trigonometry.)

In fact, one can use (for example) the foliation of each $C_{i}$ by geodesics perpendicular to $\gamma_{i}$ to obtain a Euclidean metric $\hat{\sigma}$ on $C_{i}$ which is bilipschitz equivalent to $\sigma$, with a uniform constant.

Now for each arc $\tau$ of $\beta_{1} \cap Q_{k}$, associate a point $x(\tau)$ on $\alpha_{i j}$ if the endpoints of $\tau$ lie on $\gamma_{i}$ and $\gamma_{j}$. Arrange the points $\{x(\tau)\}$ so that they are uniformly spaced on each $\alpha_{i j}$. There is a unique arc $\tau^{\prime}$, homotopic to $\tau$, which spirals in $C_{i}$ from $\gamma_{i}$ to $x(\tau)$, and then in $C_{j}$ from $x(\tau)$ to $\gamma_{j}$, and is a $\hat{\sigma}$-geodesic in each $C_{i}$. The homotopy from $\tau$ to $\tau^{\prime}$ will move no point more than a bounded distance (this is easiest to see in the universal cover - see figure 4). It is also easy to see that the resulting arcs will be disjoint

We can treat the case with cusps similarly, except that we first excise a horospherical neighborhood of each puncture with boundary length $\epsilon_{0}$, and work in the complement.

If $\beta_{1}$ has a closed component in $Q_{k}$, it must be homotopic to a boundary component $\gamma_{j}$, and no part of $\beta_{1}$ can cross that component. Thus we can take a collar neighborhood of $\gamma_{j}$ of definite width and space the corresponding components evenly (and maximally) there.

Now for maximally spaced curves in a Euclidean annulus it is clear that the spacing is approximately the area divided by the total length. Where two collars meet 
(a)

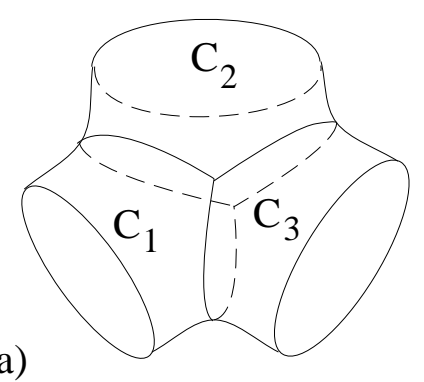

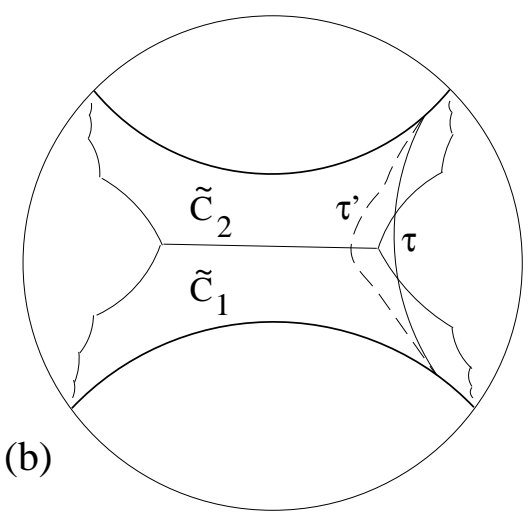

Figure 4. (a) The decomposition of a pair of pants into three annuli. (b) In the lifts of two adjacent annuli to $\mathbf{H}^{2}$, an example of an $\operatorname{arc} \tau$ and the homotopic arc $\tau^{\prime}$.

along a common boundary segment, the local picture is, up to uniform bilipschitz distortion, as in figure 5, and it is an exercise in Euclidean geometry to see that the spacing is equal to the minimum of the spacings on either side.
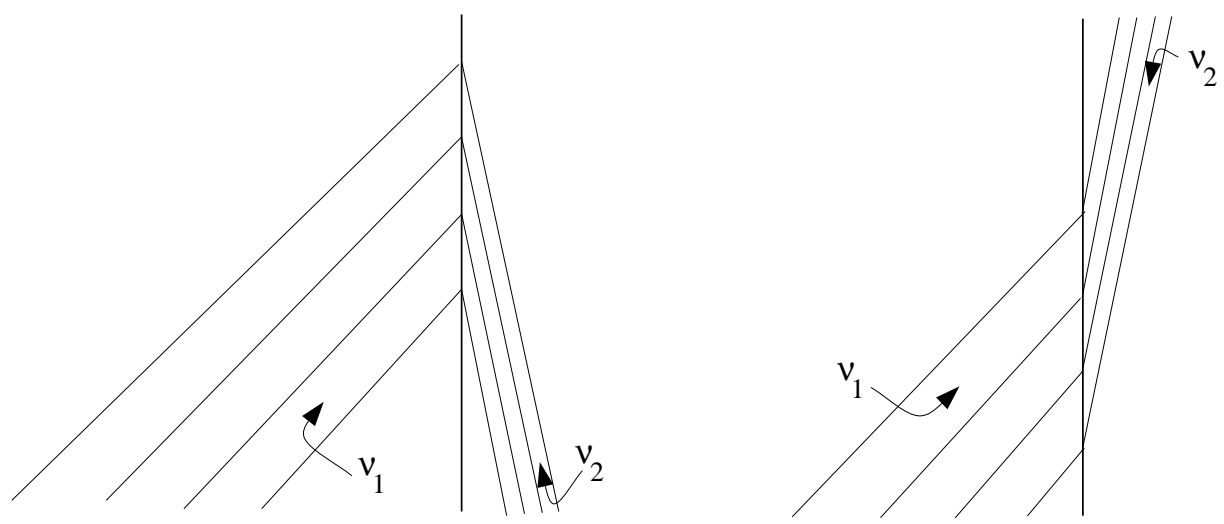

FiguRE 5

Thus we have, since the area of each $Q_{k}$ is bounded,

$$
\nu_{Q_{k}, \sigma}\left(\beta_{2} \cap Q_{k}\right) \geq \frac{C}{\ell_{\sigma}\left(\beta^{\sigma} \cap Q_{k}\right)}
$$

for each $Q_{k}$, and for all of $P$ we obtain

$$
\nu_{P, \sigma}\left(\beta_{2}\right) \geq C^{\prime} \min _{k} \nu_{Q_{k}, \sigma}\left(\beta_{2} \cap Q_{k}\right)
$$

since again the spacing is not reduced by more than a constant factor at the interfaces between subsurfaces.

Setting $\beta^{\prime}=\beta_{2}$ and observing that $\ell_{\sigma}\left(\beta^{\sigma} \cap P\right) \geq \max _{k} \ell_{\sigma}\left(\beta^{\sigma} \cap Q_{k}\right)$, the statement of lemma 4.4 follows. 
Now we can apply the spacing lemma 4.1 and the fact that the area of $P$ is bounded in terms of its Euler characteristic to conclude that

$$
\lambda_{P, \sigma}(\beta) \leq \frac{C}{\nu_{P, \sigma}^{2}\left(\beta^{\prime}\right)} \leq C \ell_{\sigma}^{2}\left(\beta^{\sigma} \cap P\right) .
$$

To obtain the inequality in the other direction we observe that the minimal length in the homotopy class $\mathcal{R}_{P}(\beta)$ is nearly attained by $\beta^{\sigma} \cap P$. In fact, the shortest representative $\beta^{P}$ can be obtained by sliding the endpoints of $\beta^{\sigma} \cap P$ around $\partial P$ until an orthogonal arc is obtained. The sliding distance is uniformly bounded by lemma 4.5 which appears below. Thus, $\ell_{\sigma}\left(\beta^{P}\right) \geq c \ell_{\sigma}\left(\beta^{\sigma} \cap P\right)$ for uniform $c$, and we conclude by the definition of extremal length that

$$
\lambda_{P, \sigma}(\beta) \geq c^{2} \frac{\ell_{\sigma}^{2}\left(\beta^{\sigma}\right)}{\operatorname{Area}(S, \sigma)} .
$$

Since $\operatorname{Area}(S, \sigma)$ is determined by the Euler characteristic of $S$, we are done with lemma 4.3 .

Lemma 4.5. Let $A_{1}$ and $A_{2}$ be annuli (possibly the same) of the $\left(\epsilon_{0}, \epsilon_{1}\right)$ collar decomposition of $(S, \sigma)$, and let $\alpha, \beta$ be geodesic arcs in $S-\left(A_{1} \cup A_{2}\right)$ with endpoints on $\partial A_{1} \cup \partial A_{2}$, such that $\alpha$ is homotopic to $\beta$ keeping the endpoints on the boundary. Then the homotopy can be taken to move the endpoints of $\alpha$ by no more than $c=$ $c\left(\epsilon_{0}, \epsilon_{1}\right)$.

Proof of 4.5: Lifting to $\widetilde{A}_{1}, \widetilde{A}_{2}, \widetilde{\alpha}$ and $\widetilde{\beta}$ in $\mathbf{H}^{2}$, we see that the endpoints of $\widetilde{\alpha}$ and $\widetilde{\beta}$ must lie in the $\operatorname{arcs} a_{1}$ and $a_{2}$ in figure 6 . It is fairly easy to see that the extremal case (longest $a_{i}$ ) occurs when $\widetilde{A}_{1}$ and $\widetilde{A}_{2}$ are actually tangent, and each is the $r$ neighborhood of a geodesic, where $r=\cosh ^{-1}\left(\epsilon_{0} / \epsilon_{1}\right)$. The length of $a_{1}$ and $a_{2}$ in this case gives the constant $c$.

$\S$ 4.3. Extremal length on annuli. For annuli $A$ in $S$ we will define a quantity $\lambda_{A, \sigma}(\beta)$ which is not exactly extremal length, but will quantify the extremal length contribution of $A$ to the whole curve $\beta \in \mathcal{C}(S, \partial S)$.

In particular, if $A$ is an annulus of the $\left(\epsilon_{0}, \epsilon_{1}\right)$ collar decomposition of $(S, \sigma)$ with core curve $\gamma$, then for $\beta \in \mathcal{C}(S, \partial S)$ let $i(\beta, A)$ or $i(\beta, \gamma)$ denote the (minimal) intersection number of $\beta$ with $\gamma$ (note, this includes the case that $A$ is peripheral, $\gamma \subset \partial S$ and $\beta$ has $i(\beta, \gamma)$ endpoints on $\gamma)$. The geodesic $\beta^{\sigma}$ can meet $A$ either in a collection of $i(\beta, A)$ properly embedded arcs, or if $i(\beta, A)=0$, in a union of closed 


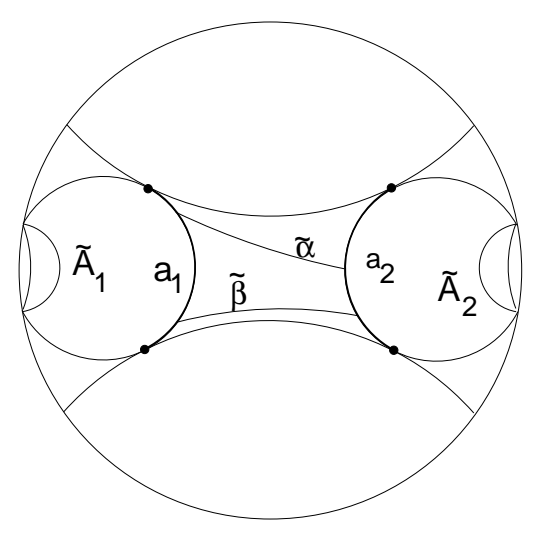

FiguRE 6

curves homotopic to $\gamma$. In either case let $n(\beta, A)$ denote the number of components of $\beta^{\sigma} \cap A$. We define

$$
\lambda_{A, \sigma}(\beta)= \begin{cases}i(\beta, A)^{2}\left(m(A)+\frac{t_{A, \sigma}(\beta)^{2}}{m(A)}\right) & \text { if } i(\beta, A)>0 \\ n(\beta, A)^{2} / m(A) & \text { otherwise. }\end{cases}
$$

It is worth nothing that, if $t_{A, \sigma}(\beta)$ is an integer and a gluing together (without twisting) of the boundaries of $A$ takes $\beta$ to a closed curve on the torus, then (4.3) gives the extremal length of the homotopy class of this curve. Compare also to lemma 2.2 .

\section{$\S 5$. The main length estimate}

For the remainder of the paper we shall fix a choice of $\epsilon_{0}>\epsilon_{1}>0$ so that the following hold for any standard hyperbolic metric $\sigma$ on $S$.

(1) $\epsilon_{0}$ is less than $\epsilon_{\mathcal{M}}$, the Margulis constant of $\mathbf{H}^{2}$.

(2) Each annulus of the $\left(\epsilon_{0}, \epsilon_{1}\right)$ collar decomposition has modulus at least 1 . It is furthermore contained in a slightly larger annulus, so that each internal boundary component bounds an annulus in the thick part of modulus $m_{0}$, where $m_{0}$ is the modulus bound in lemma 4.2.

(3) A simple geodesic in $(S, \sigma)$, if it intersects an annulus of the $\left(\epsilon_{0}, \epsilon_{1}\right)$ collar decomposition, does so either in arcs that connect the boundaries or it is the geodesic core of the annulus.

(4) Lemma 3.2 holds.

Let $\sigma$ denote a fixed standard hyperbolic metric on $S$, and let $\mathcal{Q}=\mathcal{P} \cup \mathcal{A}$ denote the $\left(\epsilon_{0}, \epsilon_{1}\right)$ collar decomposition. of section 2.4 .

Theorem 5.1. Let $S$ be a surface of finite type with a standard hyperbolic metric $\sigma$ in which boundary lengths are at most $\ell_{0}$, and let $\mathcal{Q}$ be the set of components of 
the $\left(\epsilon_{0}, \epsilon_{1}\right)$ collar decomposition. Then, for any $\beta \in \mathcal{C}(S, \partial S)$ we have

$$
\lambda_{S, \sigma}(\beta) \asymp \max _{Q \in \mathcal{Q}} \lambda_{Q, \sigma}(\beta)
$$

where the constants for $\asymp$ depend only on $\epsilon_{0}, \epsilon_{1}, \ell_{0}$ and the topological type of $S$.

Proof of 5.1: Fix, for the remainder of the section, a class $\beta \in \mathcal{C}(S, \partial S)$. Throughout the rest of the section we shall make the abbreviations $\lambda_{Q}=\lambda_{Q, \sigma}(\beta), t_{A}=t_{A, \sigma}(\beta)$, $m_{A}=m(A, \sigma)$, and $n(A)=n(\beta, A)$. On each annulus $A \in \mathcal{A}$, let $\sigma_{e}$ denote the unique standard Euclidean metric on $A$ which is conformally equivalent to $\sigma$, and agrees with $\sigma$ on $\partial A$.

The upper bound. To bound $\lambda_{S, \sigma}(\beta)$ from above, we shall need to construct a metric on $S$, in the conformal class of $\sigma$, which is sufficiently similar to the extremal metric for $\beta$ that the spacing estimate in lemma 4.1 can be applied. We remark that it is of course known that an extremal metric exists, and in fact is given by a quadratic differential. Our construction, by contrast, is not extremal (in fact we make no explicit comparison to the extremal metric), but gives concrete estimates which are explicitly related to the hyperbolic geometry of $(S, \sigma)$.

Let us make some new definitions. Let $h_{1}: \mathcal{Q} \rightarrow[0, \infty)$ be given by

$$
h_{1}(Q)= \begin{cases}\sqrt{\lambda_{Q}} & Q \in \mathcal{P} \\ \sqrt{\lambda_{Q} / m_{Q}} & Q \in \mathcal{A} .\end{cases}
$$

This quantity has the property that the scaled metrics $h_{1}(P) \sigma$ on $P \in \mathcal{P}$ and $h_{1}(A) \sigma_{e}$ on $A \in \mathcal{A}$ have areas comparable to $\lambda_{P}$ and $\lambda_{A}$.

However, in order to scale the metric in a consistent way on the entire surface we shall need to adjust $h_{1}$ to obtain a function $h$, as follows:

Lemma 5.2. There exists a positive function $h: \mathcal{Q} \rightarrow \mathbf{R}_{+}$such that the following hold.

(1) $h(Q) \geq h_{1}(Q)$ for all $Q \in \mathcal{Q}$,

(2) $\max _{Q} h(Q)=\max _{Q} h_{1}(Q)$,

and, whenever $A \in \mathcal{A}$ is adjacent to $P \in \mathcal{P}$,

(3) $h(A) \leq h(P)$.

Furthermore, for any internal annulus $A \in \mathcal{A}$ which is adjacent to components $P_{1}, P_{2} \in \mathcal{P}$ :

(4) $h(A)^{2} \geq h\left(P_{1}\right) h\left(P_{2}\right) e^{-\pi m_{A}}$, and if $A$ is peripheral and adjacent to $P \in \mathcal{P}$,

(5) $h(A) \geq h(P) e^{-\pi m_{A} / 2}$.

Proof of 5.2: Define $h_{2}(A)=h_{1}(A)$ for $A \in \mathcal{A}$. For $P \in \mathcal{P}$, let $A_{1}, \ldots, A_{k} \in \mathcal{A}$ be the annuli adjacent to $P$. Define

$$
h_{2}(P)=\max \left\{h_{1}(P), h_{1}\left(A_{1}\right), \ldots, h_{1}\left(A_{k}\right)\right\} .
$$


This function satisfies conditions (1), (2) and (3) of the lemma, and note also that $h_{2}(P)>0$ for at least one $P$. Next, let us adjust $h_{2}$ to a function $h_{n}$ that satisfies the following additional condition: for each internal $A \in \mathcal{A}$ adjacent to $P_{1}, P_{2} \in \mathcal{P}$, we require that $h\left(P_{1}\right) h\left(P_{2}\right)>0$, and

$$
\left|\log \frac{h_{n}\left(P_{1}\right)}{h_{n}\left(P_{2}\right)}\right| \leq \pi m_{A} .
$$

If the condition fails for $h_{2}$, then it must fail with some $A, P_{1}, P_{2}$ for which $h_{2}\left(P_{1}\right)>0$ and $h_{2}\left(P_{2}\right)<h_{2}\left(P_{1}\right)$. Define

$$
h_{3}\left(P_{2}\right)=h_{2}\left(P_{1}\right) e^{-\pi m_{A}},
$$

and $h_{3}=h_{2}$ elsewhere. Clearly $h_{3} \geq h_{2}$, and the maximal value of $h_{2}$ hasn't been changed. We may repeat this process to obtain a sequence of functions $h_{1}, h_{2}, h_{3}, \ldots$, which clearly terminates after a finite number of steps in a function $h_{n}$ that satisfies conditions (1),(2),(3) and (5.3). Note also that $h_{n}$ is positive on $\mathcal{P}$, since if $h_{n}(P)=0$ after these adjustments, then $P$ must be adjacent only to peripheral annuli on which $h_{1}=0$, and in that case $\beta$ is empty (we are assuming that $S$ is connected).

For an internal annulus $A \in \mathcal{A}$ adjacent to $P_{1}, P_{2}$, if $h_{n}$ fails to satisfy condition (4) we make a final adjustment:

$$
h(A)=\left(h_{n}\left(P_{1}\right) h_{n}\left(P_{2}\right) e^{-\pi m_{A}}\right)^{1 / 2} .
$$

If $A$ is peripheral, adjacent to $P$ and fails condition (5), we adjust by

$$
h(A)=h_{n}(P) e^{-\pi m_{A} / 2}
$$

We let $h=h_{n}$ for other annuli and for $P \in \mathcal{P}$. Note that $h$ still satisfies conditions (1)-(3). In particular, condition (3) is satisfied by virtue of the inequality (5.3). This concludes the proof of lemma 5.2.

The next step is to define a new metric $\sigma^{\prime}$ on $S$, conformally equivalent to $\sigma$. For $P \in \mathcal{P}$ we have simply

$$
\left.\sigma^{\prime}\right|_{P}=h(P) \sigma .
$$

For an internal annulus $A$, let $P_{1}$ and $P_{2}$ be the components of $\mathcal{P}$ which adjoin $A$. For each $P_{i}$, if $h\left(P_{i}\right)>h(A)$ we must interpolate between the natural scalings for $P_{i}$ and $A$. Let $m=m_{A}$ and let

$$
\mu_{i}=\frac{1}{2 \pi} \log \left(h\left(P_{i}\right) / h(A)\right) .
$$

This is the modulus of an annular region in the plane between two concentric circles whose radii are in the ratio $h\left(P_{i}\right) / h(A)$. We would like $\sigma^{\prime}$ to be precisely such a metric on subannuli of $A$ of appropriate modulus. In addition we want to reserve a sufficiently large (modulus at least $m / 2$ ) middle section of $A$ for other purposes. It is for these reasons that condition (4) was imposed in lemma 5.2. 
Condition (4) implies that $\mu_{1}+\mu_{2} \leq m / 2$. Thus, let $B_{1}$ and $B_{2}$ be annulus neighorhoods of the boundary components of $A$, whose radii in the Euclidean metric $\sigma_{e}$ are $\epsilon_{0} \mu_{1}, \epsilon_{0} \mu_{2}$. Let $C$ denote the closure of the complementary annulus $A-B_{1}-$ $B_{2}$; the modulus of $C$ is at least $m / 2$.

On $B_{1}$ and $B_{2}$ we can make $\sigma^{\prime}$ the metric conformally equivalent to $\sigma$ such that $\left(B_{i}, \sigma^{\prime}\right)$ is a planar annulus as above, with boundary lengths $\epsilon_{0} h\left(P_{i}\right)$ (for the boundary between $A$ and $P_{i}$ ) and $\epsilon_{0} h(A)$ on the inner boundary. On the remaining annulus $C$ between $B_{1}$ and $B_{2}$ let $\sigma^{\prime}=h(A) \sigma_{e}$. Note that this makes $\sigma^{\prime}$ a continous metric on $S$. (See figure 7.)

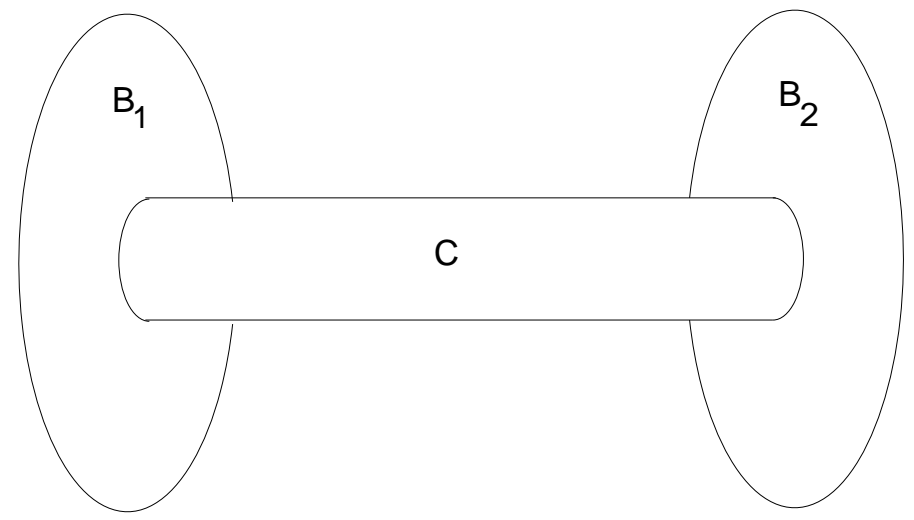

Figure 7. The metric $\sigma^{\prime}$ on the annulus $A$ decomposed into $B_{1}, C$ and $B_{2}$.

Similarly if $A$ is a peripheral annulus adjacent to $P$, we can apply condition (5) of lemma 5.2 implies that $\mu=\frac{1}{2 \pi} \log (h(P) / h(A)) \leq m / 2$. Thus we place a planar annulus metric on the annulus neighborhood $B$ of $P \cap A$, of $\sigma_{e}$-radius $\epsilon_{0} \mu$. On $A-B$ we again let $\sigma^{\prime}=h(A) \sigma_{e}$.

Finding a good representative. Having defined $\sigma^{\prime}$, we shall now construct a representative of $\beta$ whose spacing in $\sigma^{\prime}$ is as uniform as possible.

Lemma 5.3. There is a representative $\beta_{2}$ of $\beta$ whose spacing in the metric $\sigma^{\prime}$ is bounded by

$$
\nu_{S, \sigma^{\prime}}\left(\beta_{2}\right) \geq c .
$$

The constant $c$ depends only on $\epsilon_{0}, \epsilon_{1}, \ell_{0}$ and $\chi(S)$.

Proof of 5.3: We begin by applying lemma 4.4 to deform $\beta \cap P$ (if non-empty) on each $P \in \mathcal{P}$ to a curve system $\beta_{1}$ whose intersection with each boundary component of $P$ is evenly spaced, and whose spacing on the interior satisfies $\nu_{P, \sigma} \geq c / \ell_{\sigma}\left(\beta^{\sigma} \cap P\right)$. (The portions of $\beta$ in the annuli $A \in \mathcal{A}$ can be moved along with the homotopy on $P \in \mathcal{P}$, and we will examine these separately). By lemma 4.3, this implies

$$
\nu_{P, \sigma}\left(\beta_{1}\right) \geq c^{\prime} / \sqrt{\lambda_{P}}=c^{\prime} / h_{1}(P)
$$

and therefore

$$
\nu_{P, \sigma^{\prime}}\left(\beta_{1}\right) \geq c^{\prime} h(P) / h_{1}(P) \geq c^{\prime}
$$


Now consider the case of an internal annulus $A \in \mathcal{A}$, partitioned as before into boundary annuli $B_{1}, B_{2}$ and a central annulus $C$. Suppose first that $i(\beta, A)>0$. Deform $\beta_{1} \cap A$, fixing its intersection with $\partial A$, so that each resulting arc intersects $\left(B_{i}, \sigma^{\prime}\right)$ in a radial segment, and $\left(C, \sigma^{\prime}\right)$ in a $\sigma^{\prime}$-geodesic arc that twists several times around $C$. Call this new curve $\beta_{2}$. We have

$$
t_{C, \sigma^{\prime}}\left(\beta_{2} \cap C\right)=t_{A}+\delta
$$

where the error satisfies $|\delta| \leq 1$, since by lemma 4.4 the endpoints have moved at most halfway around the boundary in the homotopy from $\beta$ to $\beta_{1}$. It follows that the length of $\beta_{2} \cap C$ is, if $i(\beta, A)>0$,

$$
\begin{aligned}
\ell_{\sigma^{\prime}}\left(\beta_{2} \cap C\right) & =n(A) h(A) \epsilon_{0} \sqrt{m(C)^{2}+\left(t_{A}+\delta\right)^{2}} \\
& \leq c \epsilon_{0} h(A) \sqrt{m_{A} \lambda_{A}}
\end{aligned}
$$

Here we are using the formula (4.3) for $\lambda_{A}=\lambda_{A, \sigma}(\beta)$. Note that the error $\delta$ can be replaced by the multiplicative factor $c$ because $m_{A} \geq 1$ (by choice of $\epsilon_{0}, \epsilon_{1}$ ).

If $i(\beta, A)=0$ and $n(A)>0$ we can make $\beta_{2} \cap A$ a disjoint union of closed $\sigma^{\prime}$-geodesics uniformly spaced in $C$. Then we have:

$$
\begin{aligned}
\ell_{\sigma^{\prime}}\left(\beta_{2} \cap C\right) & =n(A) h(A) \epsilon_{0} \\
& =\epsilon_{0} h(A) \sqrt{m_{A} \lambda_{A}}
\end{aligned}
$$

again using (4.3). Now, since $\beta_{2}$ is uniformly spaced in $C$ with respect to the standard Euclidean metric $\sigma^{\prime}$, its spacing is equal to the area divided by its length. Applying either (5.10) or (5.11), and the fact that $m(C) \geq m_{A} / 2$, we obtain:

$$
\begin{aligned}
\nu_{C, \sigma^{\prime}}\left(\beta_{2} \cap C\right) & =\frac{\operatorname{Area}\left(C, \sigma^{\prime}\right)}{\ell_{\sigma^{\prime}}\left(\beta_{2} \cap C\right)} \\
& \geq c \frac{\epsilon_{0}^{2} h(A)^{2} m_{A} / 2}{\epsilon_{0} h(A) \sqrt{m_{A} \lambda_{A}}} \\
& =c^{\prime} \frac{h(A)}{h_{1}(A)} .
\end{aligned}
$$

The spacing in the boundary annuli $B_{1}, B_{2}$ is no smaller than this, and $h(A) \geq h_{1}(A)$ by lemma 5.2 , so we conclude

$$
\nu_{A} \geq c^{\prime}
$$

The case when $A$ is peripheral is dealt with in a similar way, and (5.13) still holds. In fact it is slightly easier since $t_{A}=0$ in this case.

Thus we have a curve $\beta_{2}$ with a uniform lower bound on $\nu_{Q, \sigma^{\prime}}$ for all $Q \in \mathcal{Q}$. We can now make the same observation as in the proof of lemma 4.4 to conclude that

$$
\nu_{S, \sigma^{\prime}}\left(\beta_{2}\right) \geq c \min _{Q \in \mathcal{Q}} \nu_{Q, \sigma^{\prime}}\left(\beta_{2}\right) .
$$

Namely, since on a uniformly sized neighborhood of an interface between two components the situation is uniformly bilipschitz equivalent to the Euclidean situation 
depicted in figure 5, the spacing of the combined curve is no less than a constant times the minimum of the spacings on either side. This concludes the proof of lemma 5.3.

We can now apply lemma 4.1 to obtain:

$$
\lambda_{S, \sigma}(\beta) \leq c \operatorname{Area}\left(S, \sigma^{\prime}\right)=c \sum_{Q \in \mathcal{Q}} \operatorname{Area}\left(Q, \sigma^{\prime}\right) .
$$

The desired upper bound on $\lambda_{S, \sigma}$ will be obtained if we show that

$$
\max _{Q} \operatorname{Area}\left(Q, \sigma^{\prime}\right) \leq c \max _{Q} \lambda_{Q}
$$

for a uniform constant $c$, since the number of terms in the sum (5.14) is bounded in terms of the topology of $S$.

For any $P \in \mathcal{P}$, recall that $\operatorname{Area}\left(P, \sigma^{\prime}\right) \leq c h^{2}(P)$ for a constant $c$. An internal annulus $A \in \mathcal{A}$ is divided into three subannuli $B_{1}, B_{2}$ and $C$ whose areas are $\operatorname{Area}\left(C, \sigma^{\prime}\right) \leq \epsilon_{0} h^{2}(A) m_{A}$, and $\operatorname{Area}\left(B_{i}, \sigma^{\prime}\right)=\epsilon_{0}^{2}\left(h^{2}\left(P_{i}\right)-h^{2}(A)\right) / 4 \pi$, where $P_{1}, P_{2}$ are the neighboring components of $\mathcal{P}$. A peripheral annulus is treated similarly. Thus, it suffices to bound the quantities $h^{2}(P)$ and $h^{2}(A) m_{A}$.

Note first that, by definition $(5.1), h_{1}^{2}(P) \leq \lambda_{Q}$, since $m_{A} \geq 1$ for all $A \in \mathcal{A}$. Thus we have

$$
\max _{Q \in \mathcal{Q}} h_{1}^{2}(Q) \leq \max _{Q} \lambda_{Q}
$$

It follows from this and part (2) of lemma 5.2 that, for any $P \in \mathcal{P}$,

$$
h^{2}(P) \leq \max _{Q} \lambda_{Q}
$$

Now for $A \in \mathcal{A}$, suppose first that $h(A)=h_{1}(A)$. In this case

$$
\begin{aligned}
h^{2}(A) m_{A} & =h_{1}^{2}(A) m_{A} \\
& =\lambda_{A} \leq \max _{Q} \lambda_{Q}
\end{aligned}
$$

If $h(A)>h_{1}(A)$ then, if $A$ is internal we use (5.5) in the proof of lemma 5.2 to obtain

$$
\begin{aligned}
h^{2}(A) m_{A} & =h_{n}\left(P_{1}\right) h_{n}\left(P_{2}\right) m_{A} e^{-\pi m_{A}} \\
& \leq \max _{Q} h_{1}^{2}(Q) m_{A} e^{-\pi m_{A}} \\
& \leq \frac{1}{\pi e} \max _{Q} \lambda_{Q}
\end{aligned}
$$

where the last line follows from (5.16) and the fact that $x e^{-\pi x} \leq 1 / \pi e$ for all $x$. In case $A$ is peripheral we apply (5.6), to similar effect. The bounds (5.17-5.19) 
together imply (5.15), so that with (5.14) we have the desired upper bound

$$
\lambda_{S, \sigma}(\beta) \leq c \max _{Q \in \mathcal{Q}} \lambda_{Q, \sigma}(\beta) .
$$

The lower bound. For each $Q \in \mathcal{Q}$, we shall find a metric $\sigma^{\prime \prime}$ conformal to $\sigma$ such that $\ell_{\sigma^{\prime \prime}}^{2}([\beta]) / \operatorname{Area}\left(S, \sigma^{\prime \prime}\right)$ is approximately $\lambda_{Q}$. This will give the desired lower bound.

If $Q=P \in \mathcal{P}$, let $\sigma^{\prime \prime}=\sigma$. Note that $\ell_{\sigma}([\beta]) \geq \ell_{\sigma}\left(\beta^{\sigma} \cap P\right) \asymp \sqrt{\lambda_{Q}}$, and that $\operatorname{Area}(S, \sigma)$ is a constant, $2 \pi|\chi(S)|$. Thus:

$$
\lambda_{S, \sigma}(\beta) \geq \frac{\ell_{\sigma}^{2}(\beta)}{\operatorname{Area}(S, \sigma)} \succ \lambda_{Q}
$$

If $Q=A \in \mathcal{A}$, define the metric $\sigma^{A}$ to be

$$
\sigma^{A}= \begin{cases}\sigma & \text { in } S-A \\ \sigma_{e} & \text { in } A\end{cases}
$$

where $\sigma_{e}$ is the Euclidean metric defined as in the beginning of this section.

If $i(\beta, A)=0$ but $n(A)>0$, there are $n(A)$ copies in $\beta$ of the core curve $\gamma$ of $A$. It is easy to see that $\ell_{\sigma^{A}}(\gamma)=\epsilon_{0}$ in this case, since $\sigma_{A}$ is non-positively curved so that the $\sigma_{A}$-geodesic cores of $A$ are the shortest representatives of $\gamma$. Thus,

$$
\lambda_{S, \sigma}(\beta) \geq \frac{n^{2}(A) \epsilon_{0}^{2}}{\epsilon_{0}^{2} m_{A}+2 \pi|\chi(S)|} \succ \lambda_{A} .
$$

where for the last step we use the fact that $m_{A} \geq 1$ to replace an additive constant with a multiplicative one.

Now consider the case where $i(\beta, A)>0$. Let $\beta^{A}$ be a representative of $\beta$ of minimal $\sigma^{A}$-length (in fact $\beta^{A}$ is unique, but we do not need to use this), and note that each arc of $\beta^{A} \cap(S-A)$ is geodesic, and homotopic with endpoints on $\partial A$ to a corresponding arc of $\beta^{\sigma} \cap(S-A)$ (this is most clearly seen in the universal cover). Thus by lemma 4.5 , the homotopy between $\beta^{\sigma}$ and $\beta^{A}$ only moves the intersection with $\partial A$ a bounded amount $c$. In particular we may conclude that

$$
\left|t_{A, \sigma}\left(\beta^{A}\right)-t_{A}\right| \leq 2 c / \epsilon_{0}
$$

and therefore

$$
\begin{aligned}
\ell_{\sigma^{A}}\left(\beta^{A} \cap A\right) & \geq n(A) \epsilon_{0} \sqrt{m^{2}(A)+\left(t_{A}-2 c / \epsilon_{0}\right)^{2}} \\
& \succ n(A) \epsilon_{0} \sqrt{m^{2}(A)+t_{A}^{2}} \\
& \succ \sqrt{\lambda_{A} m_{A}} .
\end{aligned}
$$

(Note that we have again used the fact that $m_{A} \geq 1$.)

We conclude that

$$
\lambda_{S, \sigma}(\beta) \succ \frac{\lambda_{A} m_{A}}{\epsilon_{0} m_{A}+2 \pi|\chi(S)|} \succ \lambda_{A} .
$$

This completes the proof of the lower bound and therefore of theorem 5.1 
We can generalize theorem 5.1 slightly as follows. Suppose that $A_{1}, \ldots, A_{k}$ are a subset of the annuli of the $\left(\epsilon_{0}, \epsilon_{1}\right)$ collar decomposition of $(S, \sigma)$ and $P_{1}, \ldots, P_{m}$ are the components of $S-\cup_{i} A_{i}$. We call $\mathcal{Q}=\left\{A_{1}, \ldots, A_{k}, P_{1}, \ldots, P_{m}\right\}$ a partial $\left(\epsilon_{0}, \epsilon_{1}\right)$ decomposition. We can then state:

Corollary 5.4. If $\mathcal{Q}$ is a partial $\left(\epsilon_{0}, \epsilon_{1}\right)$ collar decomposition with $\epsilon_{0}, \epsilon_{1}$ chosen as above, then for any $\beta \in \mathcal{C}(S, \partial S)$

$$
\lambda_{S, \sigma} \asymp \max _{Q \in \mathcal{Q}} \lambda_{Q, \sigma}(\beta) .
$$

Proof of 5.4: Let $\overline{\mathcal{Q}}=\overline{\mathcal{P}} \cup \overline{\mathcal{A}}$ be the full $\left(\epsilon_{0}, \epsilon_{1}\right)$ collar decomposition. Let $\overline{\mathcal{Q}}_{i}=\overline{\mathcal{P}}_{i} \cup \overline{\mathcal{A}}_{i}$ be the subset of $\overline{\mathcal{Q}}$ consisting of components that lie in $P_{i}$. Let $\hat{\sigma}_{i}$ be the standard hyperbolic metric on $P_{i}$ which is conformally equivalent to the restriction of $\sigma$. We first show that, for any $\bar{Q} \in \overline{\mathcal{Q}}_{i}$,

$$
\lambda_{\bar{Q}, \sigma}(\beta) \asymp \lambda_{\bar{Q}, \hat{\sigma}}\left(\mathcal{R}_{P_{i}}(\beta)\right) .
$$

For a hyperbolic component $\bar{Q} \in \overline{\mathcal{P}}_{i}$, these two quantities are the same by definition (4.2), since only the conformal class of the metric matters for extremal length.

For an annulus $\bar{Q}=\bar{A} \in \overline{\mathcal{A}}_{i}$, the definition (4.3) depends in particular on the measure of twisting $t_{\bar{A}, \sigma}$, which depends on the actual geometry. However, lemma 3.4 assures us that the difference between twisting in $\sigma$ and $\hat{\sigma}$ is no more than a constant. It follows that (5.24) holds for annuli as well, with uniform constants that depend only on $\epsilon_{0}, \epsilon_{1}$ and the topology of $S$.

The corollary now follows by applying theorem 5.1 separately to each $P_{i}$.

\section{$\S 6$. The product region theorem}

In this section $S$ denotes an oriented finite genus surface without boundary, but possibly with finitely many punctures.

Let $\gamma$ be an element of $\mathcal{C}(S)$ all of whose components $\gamma_{1}, \ldots, \gamma_{k}$ are homotopically distinct, and fix an orientation on each component of $\gamma$. For any $\epsilon>0$, denote by $\operatorname{Thin}_{\epsilon}(S, \gamma)$ the subset of $\mathcal{T}(S)$ consisting of all $[\sigma]$ such that $\ell_{\sigma}\left(\gamma_{i}\right) \leq \epsilon$.

Let $\gamma_{k+1}, \ldots, \gamma_{n}$ denote a completion of $\gamma$ to an oriented pair-of-pants decomposition $\hat{\gamma}$. Fix also a curve system $\mu$ composed of seam arcs, giving a marking of $S$ as in $\S 3$. This gives rise to length and twist coordinates on $\mathcal{T}(S)$, denoted in $\S 3$ by the map $F: \mathcal{T}(S) \rightarrow \mathbf{R}_{+}^{n} \times \mathbf{R}^{n}$. Let us write the coordinates of $F$ as $F_{i}$, where $F_{1}, \ldots, F_{n}$ are length coordinates, and $F_{n+1}, \ldots, F_{2 n}$ are twist coordinates. Separating out the coordinates of the first $k$ curves from the rest decomposes $\mathcal{T}(S)$ as a product.

Let $S_{\gamma}$ denote the punctured (or "noded") surface obtained from $S$ by deleting $\gamma_{1}, \ldots, \gamma_{k}$ and replacing each curve by a pair of punctures. Let $\mathcal{T}\left(S_{\gamma}\right)$ be the space 
of (analytically finite) conformal structures on $S_{\gamma}$. Let $\mathbf{H}$ denote the upper halfplane $\{(x, y): y>0\}$ endowed with the hyperbolic metric $d_{\mathbf{H}}$. Let $X_{\gamma}$ denote the product space $X_{\gamma}=\mathcal{T}\left(S_{\gamma}\right) \times \mathbf{H}_{1} \times \cdots \times \mathbf{H}_{k}$ where each $\mathbf{H}_{i}$ is a copy of $\mathbf{H}$, endowed with the sup metric $d_{X}=\max \left(d_{\mathcal{T}\left(S_{\gamma}\right)}, d_{\mathbf{H}_{1}}, \ldots, d_{\mathbf{H}_{k}}\right)$.

The marking by $\hat{\gamma}$ and $\mu$ induces a marking on each component of $S_{\gamma}$, and a map $G: \mathcal{T}\left(S_{\gamma}\right) \rightarrow \mathbf{R}_{+}^{n-k} \times \mathbf{R}^{n-k}$, given by $G(\rho)=\left(\ell_{\rho}\left(\gamma_{k+1}\right), \ldots, \ell_{\rho}\left(\gamma_{n}\right), s_{k+1}(\rho), \ldots, s_{n}(\rho)\right)$ for any complete hyperbolic metric $\rho$ on $S_{\gamma}$.

We can therefore define a map

$$
\Pi: \mathcal{T}(S) \rightarrow \mathcal{T}\left(S_{\gamma}\right) \times \mathbf{H}_{1} \times \cdots \times \mathbf{H}_{k}
$$

as follows. Let $\Pi_{0}$ denote the component of $\Pi$ mapping to $\mathcal{T}\left(S_{\gamma}\right)$, and for $i>0$ let $\Pi_{i}$ denote the component mapping to $\mathbf{H}_{i}$. We define $\Pi_{0}$ via

$$
\Pi_{0}(\sigma)=G^{-1}\left(F_{k+1}(\sigma), \ldots, F_{n}(\sigma), F_{n+k+1}(\sigma), \ldots, F_{2 n}(\sigma)\right) .
$$

In other words, we build a punctured surface by forgetting the length and twist coordinates of the first $k$ curves, and using the remaining coordinates to build new pairs of pants. We then use the forgotten coordinates for the rest of $\Pi$, by defining

$$
\Pi_{i}(\sigma)=\left(s_{i}(\sigma), 1 / \ell_{\sigma}\left(\gamma_{i}\right)\right)
$$

for $i=1, \ldots, k$.

Let $\epsilon_{0}$ and $\epsilon_{1}$ be chosen as in the previous section. Our main theorem is the following.

Theorem 6.1. Let $\gamma \in \mathcal{C}(S)$ be an oriented curve system as above, extended to a marking $\hat{\gamma}, \mu$ of $S$. Given $\epsilon \in\left(0, \epsilon_{1}\right)$, there is a contant $a_{0}$ depending on $\epsilon_{0}, \epsilon_{1}$ and the topological type of $S$, such that the homeomorphism $\Pi: \mathcal{T}(S) \rightarrow X_{\gamma}$ defined above, when restricted to $\operatorname{Thin}_{\epsilon}(S, \gamma)$, satisfies

$$
\left|d_{\mathcal{T}(S)}(\sigma, \tau)-d_{X}(\Pi(\sigma), \Pi(\tau))\right| \leq a_{0}
$$

for any $[\sigma],[\tau] \in \operatorname{Thin}_{\epsilon}(S, \gamma)$.

Proof of 6.1: For a given $[\sigma] \in \operatorname{Thin}_{\epsilon}(S, \gamma)$, let $\sigma$ denote its hyperbolic representative. Let $A_{1}, \ldots, A_{k}$ be the annuli of the $\left(\epsilon_{0}, \epsilon_{1}\right)$ collar decomposition which correspond to the curves $\gamma_{1}, \ldots, \gamma_{k}$. The complement $S-\cup A_{i}$ is also naturally identified with $S_{\gamma}$. Let $P_{1}, \ldots, P_{m}$ denote the connected components of $S_{\gamma}$, and let $\mathcal{Q}=\left\{A_{j}\right\} \cup\left\{P_{i}\right\}$. Note that for another point $[\tau] \in \operatorname{Thin}_{\epsilon}(S, \gamma)$ we obtain a similar decomposition, which we also call $\mathcal{Q}$.

Our strategy will be to estimate distances using Kerckhoff's theorem, so we shall need to be able to control extremal length ratios of the type $\lambda_{Q, \sigma}(\alpha) / \lambda_{Q, \tau}(\alpha)$. Note that, for $Q$ a subsurface of $S$, if $\lambda_{Q, \sigma}(\alpha)=0$ then $\lambda_{Q, \tau}(\alpha)=0$ as well. Thus we adopt the convention that, if $\lambda_{Q, \sigma}(\alpha)=0$ then the expression $\lambda_{Q, \sigma}(\alpha) / \lambda_{Q, \tau}(\alpha)$ is defined to be 1 .

The first step is the following: 
Lemma 6.2. Let $Q$ be any hyperbolic surface of finite type with boundary, and $\lambda_{1}>\lambda_{0}>0$ two given constants. Let $[\sigma],[\tau] \in \mathcal{T}(Q)$ be two conformal structures such that $\lambda_{Q, \sigma}(\gamma) \in\left[\lambda_{0}, \lambda_{1}\right]$ for any boundary component $\gamma$, and similarly for $\tau$. Then

$$
\sup _{\alpha \in \mathcal{C}_{0}(Q)} \frac{\lambda_{Q, \sigma}(\alpha)}{\lambda_{Q, \tau}(\alpha)} \asymp \sup _{\alpha \in \mathcal{C}(Q, \partial Q)} \frac{\lambda_{Q, \sigma}(\alpha)}{\lambda_{Q, \tau}(\alpha)}
$$

where the constants for “ $\asymp$ " depend only on $\chi(S), \lambda_{0}$ and $\lambda_{1}$.

Proof of 6.2: The left side is clearly no larger than the right, since $\mathcal{C}_{0}(Q) \subset \mathcal{C}(Q, \partial Q)$.

The supremum on the right side is equal to $\exp 2 d_{\mathcal{T}(Q)}(\sigma, \tau)$, by Kerckhoff's theorem 2.1, where $d_{\mathcal{T}(Q)}$ is the distance in the Teichmüller space of $Q$. In particular, this distance is symmetric, so that the supremum of the reciprocal ratio is the same quantity. Choose $\beta \in \mathcal{C}(Q, \partial Q)$ such that $\lambda_{Q, \tau}(\beta) / \lambda_{Q, \sigma}(\beta)$ is nearly equal to the supremum on the right side of (6.4).

By lemma 6.3, whose proof appears below, there exists $\alpha \in \mathcal{C}_{0}(S)$ such that, for a uniform $c>0$,

$$
i(\alpha, \beta)^{2} \geq c \lambda_{Q, \tau}(\alpha) \lambda_{Q, \tau}(\beta) .
$$

(One should think of $\alpha$ as approximately "orthogonal" to $\beta$.)

On the other hand, the following inequality is elementary for any two curves $\alpha, \beta \in \mathcal{C}(Q)$ and $[\sigma] \in \mathcal{T}(Q)$ (see e.g. [23, Lemma 5.1]).

$$
\lambda_{Q, \sigma}(\alpha) \lambda_{Q, \sigma}(\beta) \geq i(\alpha, \beta)^{2} .
$$

Combining (6.5) with(6.6), we obtain

$$
\frac{\lambda_{Q, \sigma}(\alpha)}{\lambda_{Q, \tau}(\alpha)} \geq c \frac{\lambda_{Q, \tau}(\beta)}{\lambda_{Q, \sigma}(\beta)}
$$

which gives the remaining direction of lemma 6.2 .

To complete lemma 6.2 we need to prove the following:

Lemma 6.3. Let $Q$ be a hyperbolic surface of finite type, with a conformal structure $[\tau]$. Suppose that for $0<\lambda_{0}<\lambda_{1}$ we have $\lambda_{Q, \tau}(\gamma) \in\left[\lambda_{0}, \lambda_{1}\right]$ for any boundary component $\gamma$ of $Q$. Then for any $\beta \in \mathcal{C}(Q, \partial Q)$ there exists $\alpha \in \mathcal{C}_{0}(Q)$ for which

$$
i(\alpha, \beta)^{2} \geq c \lambda_{Q, \tau}(\alpha) \lambda_{Q, \tau}(\beta)
$$

where $c$ depends only on $\lambda_{0}, \lambda_{1}$ and $\chi(Q)$.

Proof of 6.3: Represent $[\tau]$ by a standard hyperbolic metric $\tau$, and let $T_{1}, \ldots, T_{j}$ be the $\left(\epsilon_{0}, \epsilon_{1}\right)$ collar decomposition of $Q$, with respect to $\tau$. Note that $\epsilon_{1}$ may be chosen (depending on $\lambda_{0}$ ) so that no annuli in the decomposition are peripheral in $Q$. Applying theorem 5.1, there exists one component, say $T_{1}$, such that $\lambda_{Q, \tau}(\beta) \asymp$ $\lambda_{T_{1}, \tau}(\beta)$. 
Suppose first that $T_{1}$ is a non-annular component. We claim that there exist constants $c_{0}, \lambda_{2}$ such that there is an $\alpha \in \mathcal{C}_{0}\left(T_{1}\right)$ with $\lambda_{T_{1}, \tau}(\alpha) \leq \lambda_{2}$, and $i(\alpha, \beta)^{2} \geq c_{0} \lambda_{T_{1}, \tau}(\beta)$. We can see this as follows: Since $T_{1}$ has no thin parts we can cut it along moderate-length curves to obtain bounded-diameter pieces. More precisely, note that the components of $\partial T_{1}$ are either boundaries of thin parts of length $\epsilon_{0}$, or boundaries of $Q$, which have $\tau$-length in some interval $\left[\ell_{0}, \ell_{1}\right]$ (depending on $\lambda_{0}, \lambda_{1}$ ). We can find a pair-of-pants decomposition for $T_{1}$ of minimal total boundary lengths, and then add sufficiently many simple closed geodesics, as short as possible, to cut $T_{1}$ into disks and boundary-parallel annuli. The result is a collection of non-peripheral simple closed geodesics $\alpha_{1}, \ldots, \alpha_{q}$ in $T_{1}$ ( $q$ dependent only on $\chi\left(T_{1}\right)$ ) such that $\ell_{\tau}\left(\alpha_{i}\right) \leq \ell_{2}$ (depending on $\epsilon_{0}, \lambda_{0}, \lambda_{1}, \chi\left(T_{1}\right)$ ) and which cut $T_{1}$ up into components that are either disks, or annuli which share a boundary with $T_{1}$, or annular neighborhoods of cusps. In the first two cases the diameters of the components are uniformly bounded. In the third case we may remove a neighborhood of the cusp avoided by $\beta$ (since $\beta$ is simple) and so that what remains has bounded diameter. Furthermore note that any component of the intersection of the geodesic $\beta^{\tau}$ with one of these regions has length at most some $\delta_{0}$, since $\beta^{\tau}$ meets $\partial Q$ orthogonally, and meets the other components of $\partial T_{1}$ nearly orthogonally (compare lemma 4.5).

It follows, adding over the pieces of $\beta^{\tau}$ in these components, that $\sum_{j} \delta_{0} i\left(\beta, \alpha_{j}\right) \geq$ $\ell_{\tau}\left(\beta^{\tau} \cap T_{1}\right)$. By lemma 4.3, $\ell_{\tau}^{2}\left(\beta^{\tau} \cap T_{1}\right) \asymp \lambda_{T_{1}, \tau}(\beta)$. Thus, there must be some $\alpha=\alpha_{j}$ such that

$$
i(\alpha, \beta)^{2} \geq C \lambda_{T_{1}, \tau}(\beta) .
$$

We also know that there is an upper bound

$$
\lambda_{Q, \tau}(\alpha) \leq \lambda_{3}
$$

for a uniform $\lambda_{3}$, since there is an upper bound on the hyperbolic length of $\alpha$ by construction. The statement of the lemma follows in this case.

Now let $T_{1}=A$ be an annular component with modulus $m=m(A, \tau)$, and consider first the case when $i(\beta, A)>0$, and $t_{1}=t_{A, \tau}(\beta) \neq 0$. Let $t_{2}=-m^{2} / t_{1}$ - we choose this number because two geodesics with twists $t_{1}, t_{2}$ in a standard Euclidean annulus of modulus $m$ are orthogonal when $t_{1} t_{2}=-m^{2}$. Lemma 3.3 guarantees the existence of an element $\alpha \in \mathcal{C}_{0}(Q)$ with $\left|t_{A, \tau}(\alpha)-t_{2}\right| \leq c_{1}$ for a uniform $c_{1}$.

Now using the definition of $\lambda_{A, \tau}$ in (4.3), we can see that

$$
\begin{aligned}
\lambda_{A, \tau}(\alpha) \lambda_{A, \tau}(\beta) & \asymp n(\alpha, A) n(\beta, A)\left(m+t_{1}^{2} / m\right)\left(m+t_{2}^{2} / m\right) \\
& =n(\alpha, A) n(\beta, A)\left(t_{1}-t_{2}\right)^{2} .
\end{aligned}
$$

where the additive error $c_{1}$ becomes a multiplicative error since $m \geq 1$.

On the other hand it is easy to see that, if two properly embedded $\operatorname{arcs} \alpha_{1}, \alpha_{2}$ in $A$ have twisting numbers $t_{i}=t_{A, \tau}\left(\alpha_{i}\right)$, then their intersection number is estimated by

$$
\left|t_{1}-t_{2}\right|-1 \leq i\left(\alpha_{1}, \alpha_{2}\right) \leq\left|t_{1}-t_{2}\right|+1
$$


Combining (6.8) with (6.7) we obtain

$$
\lambda_{A, \tau}(\alpha) \lambda_{A, \tau}(\beta) \succ i(\alpha, \beta)^{2}
$$

(again an additive error has become multiplicative). By lemma 3.3, $\alpha$ may be chosen to have bounded length outside $A$, and thus by theorem 5.1, $\lambda_{A, \tau}(\alpha) \asymp \lambda_{Q, \tau}(\alpha)$. Since also $\lambda_{A, \tau}(\beta) \asymp \lambda_{Q, \tau}(\beta)$ by choice of $A$, the statement of lemma 6.3 follows.

We have left the case where $t_{1}=0$, and the case where $i(\beta, A)=0$ but $n(\beta, A)>$ 0 . In the former case, We can pick $\alpha$ to be the core of $A$ (essentially this is $t_{2}=\infty$ ), and note that $\lambda_{Q, \sigma}(\alpha) \asymp 1 / m$, so that the lemma holds automatically. In the latter case we may choose $\alpha$ with $t_{2}=0$, and the roles of $\alpha$ and $\beta$ are reversed.

We now resume the proof of theorem 6.1. Let us establish the following for $[\sigma],[\tau] \in \operatorname{Thin}_{\epsilon}(S, \gamma):$

$$
\sup _{\alpha \in \mathcal{C}(S)} \frac{\lambda_{S, \sigma}(\alpha)}{\lambda_{S, \tau}(\alpha)} \asymp \max _{Q \in \mathcal{Q}} \sup _{\alpha \in \mathcal{C}(S)} \frac{\lambda_{Q, \sigma}(\alpha)}{\lambda_{Q, \tau}(\alpha)} .
$$

One direction is a direct application of the main extremal length estimate. Note that $\mathcal{Q}$ is what we called a "partial $\left(\epsilon_{0}, \epsilon_{1}\right)$ collar decomposition" in the previous section. Thus by corollary 5.4 of theorem 5.1 , we have

$$
\lambda_{S, \sigma}(\alpha) \asymp \max _{Q \in \mathcal{Q}} \lambda_{Q, \sigma}(\alpha)
$$

for each $\alpha \in \mathcal{C}(S)$. Letting $Q_{\alpha}$ maximize $\lambda_{Q, \sigma}(\alpha)$ over all $Q \in \mathcal{Q}$, we apply (6.11) to obtain

$$
\begin{aligned}
\sup _{\alpha \in \mathcal{C}(S)} \frac{\lambda_{S, \sigma}(\alpha)}{\lambda_{S, \tau}(\alpha)} & \asymp \sup _{\alpha \in \mathcal{C}(S)} \frac{\max _{Q \in \mathcal{Q}} \lambda_{Q, \sigma}(\alpha)}{\max _{Q \in \mathcal{Q}} \lambda_{Q, \tau}(\alpha)} \\
& \leq \sup _{\alpha \in \mathcal{C}(S)} \frac{\lambda_{Q_{\alpha}, \sigma}(\alpha)}{\lambda_{Q_{\alpha}, \tau}(\alpha)} \\
& \leq \max _{Q \in \mathcal{Q}} \sup _{\alpha \in \mathcal{C}(S)} \frac{\lambda_{Q, \sigma}(\alpha)}{\lambda_{Q, \tau}(\alpha)}
\end{aligned}
$$

For the inequality in the other direction, consider first a non-annular component $Q \in \mathcal{Q}$. By definition (4.2) of $\lambda_{Q, \sigma}$ the supremum $\sup _{\alpha \in \mathcal{C}(S)} \lambda_{Q, \sigma}(\alpha) / \lambda_{Q, \tau}(\alpha)$ may be obtained by letting $\alpha$ vary over $\mathcal{C}(Q, \partial Q)$. By lemma 6.2 , we may further restrict to $\alpha \in \mathcal{C}_{0}(Q)$, at the expense of a bounded factor. By lemma 4.2, $\lambda_{Q, \sigma}(\alpha) \asymp \lambda_{S, \sigma}(\alpha)$ for $\alpha \in \mathcal{C}_{0}(Q)$ and any $\sigma \in \operatorname{Thin}_{\epsilon}(S, \gamma)$. Since $\mathcal{C}_{0}(Q) \subset \mathcal{C}(S)$, the supremal ratio over all of $\mathcal{C}(S)$ can only be bigger, so in this case we have the desired inequality

$$
\sup _{\alpha \in \mathcal{C}(S)} \frac{\lambda_{S, \sigma}(\alpha)}{\lambda_{S, \tau}(\alpha)} \geq \sup _{\alpha \in \mathcal{C}(S)} \frac{\lambda_{Q, \sigma}(\alpha)}{\lambda_{Q, \tau}(\alpha)} .
$$


Now consider an annulus $A \in \mathcal{Q}$, and let $\alpha$ be a curve nearly maximizing the ratio $\lambda_{A, \sigma}(\alpha) / \lambda_{A, \tau}(\alpha)$. By lemma 3.3, there exists $\alpha^{\prime} \in \mathcal{C}(S)$ such that

$$
\left|t_{A, \tau}\left(\alpha^{\prime}\right)-t_{A, \tau}(\alpha)\right| \leq c_{1},
$$

and such that (applying theorem 5.1)

$$
\lambda_{S, \tau}\left(\alpha^{\prime}\right) \asymp \lambda_{A, \tau}\left(\alpha^{\prime}\right) .
$$

Applying lemmas 3.2 and 3.5, we see that $t_{A, \tau}\left(\alpha^{\prime}\right)-t_{A, \sigma}\left(\alpha^{\prime}\right)$ and $t_{A, \tau}(\alpha)-t_{A, \sigma}(\alpha)$ are both approximated, up to bounded additive error, by the twist-parameter difference $s_{j}(\tau)-s_{j}(\sigma)$ associated to $A$. This together with inequality (6.14) implies that $\left|t_{A, \sigma}\left(\alpha^{\prime}\right)-t_{A, \sigma}(\alpha)\right|$ is uniformly bounded as well. Since $\lambda_{A, \sigma}$ and $\lambda_{A, \tau}$ are determined by the twisting numbers, we conclude that

$$
\frac{\lambda_{A, \sigma}\left(\alpha^{\prime}\right)}{\lambda_{A, \tau}\left(\alpha^{\prime}\right)} \asymp \frac{\lambda_{A, \sigma}(\alpha)}{\lambda_{A, \tau}(\alpha)} .
$$

Now we may apply (6.15) together with the inequality $\lambda_{A, \sigma}\left(\alpha^{\prime}\right) \prec \lambda_{S, \sigma}\left(\alpha^{\prime}\right)$, which is a consequence of theorem 5.1, to conclude that

$$
\frac{\lambda_{S, \sigma}\left(\alpha^{\prime}\right)}{\lambda_{S, \tau}\left(\alpha^{\prime}\right)} \succ \frac{\lambda_{A, \sigma}\left(\alpha^{\prime}\right)}{\lambda_{A, \tau}\left(\alpha^{\prime}\right)} .
$$

Inequalities (6.12), (6.13) and (6.16) together imply (6.10).

The length ratio on the left side of (6.10) is, by Kerckhoff's theorem 2.1, equal to $\exp 2 d_{\mathcal{T}(S)}(\sigma, \tau)$. It remains to connect the length ratios on the right of (6.10) to the distances $d_{i}(i=0, \ldots, k)$, so that we may complete the proof of theorem 6.1.

Consider first $d_{0}$, the Teichmüller distance on $\mathcal{T}\left(S_{\gamma}\right)$. Given a $Q \in \mathcal{Q}$ which is a component of $S_{\gamma}$, with the conformal structure $\Pi_{0}(\sigma)$ or $\Pi_{0}(\tau)$ (restricted from $S_{\gamma}$ to $Q$ ), we have by Kerckhoff's theorem:

$$
\sup _{\alpha \in \mathcal{C}_{0}(Q)} \frac{\lambda_{Q, \Pi_{0}(\sigma)}(\alpha)}{\lambda_{Q, \Pi_{0}(\tau)}(\alpha)}=\exp 2 d_{\mathcal{T}(Q)}\left(\Pi_{0}(\sigma), \Pi_{0}(\tau)\right) .
$$

We now claim that the extremal length ratio on the left can be approximated by a ratio taken with respect to $\sigma$ and $\tau$. In fact we claim that $(Q, \sigma)$ embeds $K$-quasiconformally, with uniform $K$, in $\left(Q, \Pi_{0}(\sigma)\right)$. Recall the construction: we measure the length and twist parameters for $\gamma_{k+1}, \ldots, \gamma_{n}$ for $\sigma$, and then construct $\Pi_{0}(\sigma)$ with the same parameters, but with the boundary curves $\gamma_{1}, \ldots, \gamma_{k}$ replaced by punctures. Thus the embedding of $(Q, \sigma)$ in $\left(Q, \Pi_{0}(\sigma)\right)$ can be done separately on each pair of pants. After splitting each pair of pants along seam curves into rightangled hexagons, the problem reduces to the following: Let $H_{1}$ be a hyperbolic right-angled hexagon with lengths $\ell_{1}, \ell_{2}, \ell_{3}$ on alternating sides, where $\ell_{1} \leq \epsilon_{1}$, and let $H_{2}$ be a hyperbolic right-angled hexagon with lengths $0, \ell_{2}, \ell_{3}$ (really a rightangled pentagon with one ideal vertex). There is a $K$-quasiconformal embedding (in fact bilipschitz) of $H_{1}$ into $H_{2}$ which is an isometry on sides 2 and 3 , where $K$ depends on $\epsilon_{1}$, but not on the $\ell_{i}$. This fact follows easily from the hexagon cosine law (see e.g. [3]). A similar computation applies when a pair of pants has more 
than one short boundary which is to be replaced by punctures. The maps on the pairs of pants fit together across the boundaries, because the marking and twist parameters are the same for both surfaces.

It now follows (applying also lemma 4.2) that $\lambda_{Q, \sigma}(\alpha) \asymp \lambda_{Q, \Pi_{0}(\sigma)}(\alpha)$ uniformly for all $\sigma \in \operatorname{Thin}_{\epsilon}(S, \gamma)$ and $\alpha \in \mathcal{C}_{0}(Q)$. Thus

$$
\sup _{\alpha \in \mathcal{C}_{0}(Q)} \frac{\lambda_{Q, \Pi_{0}(\sigma)}(\alpha)}{\lambda_{Q, \Pi_{0}(\tau)}(\alpha)} \asymp \sup _{\alpha \in \mathcal{C}_{0}(Q)} \frac{\lambda_{Q, \sigma}(\alpha)}{\lambda_{Q, \tau}(\alpha)} .
$$

Applying again lemma 6.2, we conclude

$$
\exp 2 d_{\mathcal{T}(Q)}\left(\Pi_{0}(\sigma), \Pi_{0}(\tau)\right) \asymp \sup _{\alpha \in \mathcal{C}(Q)} \frac{\lambda_{Q, \sigma}(\alpha)}{\lambda_{Q, \tau}(\alpha)} .
$$

Since $d_{0}=d_{\mathcal{T}\left(S_{\gamma}\right)}$ is the supremum of the distances in the Teichmüller spaces of the components, we have shown that the contribution to the right side of (6.10) coming from the non-annular components is, up to bounded factor, just $\exp 2 d_{0}$.

We next consider an annular component $A_{j}$ in $\mathcal{Q}$, and estimate its extremal length contribution $\lambda_{A_{j}, \sigma}(\alpha)$, for $\alpha \in \mathcal{C}(S)$ and $\sigma \in \operatorname{Thin}_{\epsilon}(S, \gamma)$, in terms of the coordinates $\Pi_{j}(\sigma)=\left(s_{j}(\sigma), 1 / \ell_{\sigma}\left(\gamma_{j}\right)\right)$. Rewrite $\Pi_{j}(\sigma)=\left(x_{j}(\sigma), y_{j}(\sigma)\right)$, and note first that (by (2.2)) $y_{j}(\sigma)$ is closely approximated by $m\left(A_{j}, \sigma\right) / \pi$.

Fix some arbitrary $\sigma_{0} \in \operatorname{Thin}_{\epsilon}(S, \gamma)$ and define $b_{j}(\alpha)=t_{\gamma_{j}, \sigma_{0}}(\alpha)-s_{j}\left(\sigma_{0}\right)$. By lemma 3.5, $t_{\gamma_{j}, \sigma}(\alpha)$ is estimated by $b_{j}(\alpha)+s_{j}(\sigma)$, up to bounded additive error, for all $\sigma$ and $\alpha$. Applying also lemma 3.2, this gives an estimate for $t_{A_{j}, \sigma}(\alpha)$. Now applying definition (4.3) when $i\left(\alpha, \gamma_{j}\right)>0$, we obtain this approximation for the length ratio contribution of the annulus:

$$
\frac{\lambda_{A_{j}, \sigma}(\alpha)}{\lambda_{A_{j}, \tau}(\alpha)} \asymp \frac{y_{j}(\sigma)+\left(b_{j}(\alpha)+x_{j}(\sigma)\right)^{2} / y_{j}(\sigma)}{y_{j}(\tau)+\left(b_{j}(\alpha)+x_{j}(\tau)\right)^{2} / y_{j}(\tau)},
$$

Note that the case where $i\left(\alpha, \gamma_{j}\right)=0$ and $n\left(\alpha, \gamma_{j}\right)>0$ can be obtained by the limit of (6.17) as $b_{j} \rightarrow \infty$.

By lemma 3.3, we know that $t_{\gamma_{j}, \sigma_{0}}(\alpha)$, and therefore $b_{j}(\alpha)$, can take on any value in $\mathbf{R}$ up to bounded difference. Since $y_{j}(\sigma), y_{j}(\tau)$ are at least $1 / \epsilon$, we may once again convert the additive error to a multiplicative one. Thus, taking a supremum of (6.17) over all $\alpha \in \mathcal{C}(S)$, and applying lemma 2.2, we obtain

$$
\sup _{\alpha \in \mathcal{C}(S)} \frac{\lambda_{A_{j}, \sigma}(\alpha)}{\lambda_{A_{j}, \tau}(\alpha)} \asymp \exp 2 d_{\mathbf{H}_{j}}\left(\Pi_{j}(\sigma), \Pi_{j}(\tau)\right) .
$$

It follows that the right side of (6.10) is, up to bounded multiple,

$$
\max _{j=0, \ldots, k} \exp 2 d_{j}\left(\Pi_{j}(\sigma), \Pi_{j}(\tau)\right) .
$$

Taking logarithms, we obtain the desired statement of theorem 6.1. 


\section{$\S 7$. Geometric remarks}

Having shown that regions of the form $\operatorname{Thin}_{\epsilon}(S, \gamma)$ in $\mathcal{T}(S)$ are approximated up to bounded additive distortion by products with the sup metric, we discuss briefly here the implications to "non-hyperbolic" behavior of the Teichmüller space. Indeed, a product with the sup metric is not just non-negatively curved, but exhibits some definite positive-curvature behavior, as we shall see.

Let $X$ be a metric space, and let $x, y, z \in X$ denote three points. We say that $z$ is " $\delta$-between" $x$ and $y$ if

$$
d(x, z)+d(z, y)-d(x, y)<\delta
$$

for $\delta>0$. In particular if $X$ is a length space and $[x y]$ denotes a shortest path (geodesic) between $x$ and $y$, then when $z$ is $\delta$-between $x$ and $y$, the path $[x z] \cup[z y]$ is a quasigeodesic with only additive distortion $\delta$.

In a negatively curved length space in the sense of Gromov and Cannon, if $z$ is $\delta$-between $x$ and $y$ then it is contained in an $R(\delta)$-neighborhood of [xy], where $R(\delta)$ is independent of $x$ and $y$. This is called stability of quasi-geodesics.

Let us define an "instability function" $s$ for $X$ as follows: For $\delta, L \geq 0$ define $s(\delta, L)$ by

$$
s(\delta, L)=\sup \{d(z,[x y]): z \text { is } \delta \text {-between } x \text { and } y \text { and } d(x, y) \leq L\} .
$$

In order to consider only the large-scale behavior, let us denote by $\bar{s}(\delta)$ the asymptotic growth rate of $s(\delta, L)$; that is,

$$
\bar{s}(\delta)=\limsup _{L \rightarrow \infty} \frac{\log s(\delta, L)}{\log L} .
$$

It is easy to see that $\bar{s}(\delta) \in[0,1]$, and $\bar{s}$ is a non-decreasing function of $\delta$.

In a negatively curved space, $s(\delta, L)$ is independent of $L$ for large $L$. Thus, $\bar{s} \equiv 0$. In Euclidean space it is not hard to see that $s(\delta, L)=\sqrt{2 L \delta+\delta^{2}} / 2$. In particular $\bar{s}(\delta)=1 / 2$ for $\delta>0$. In a space which contains large positively curved regions such as hemispheres, $s$ could be made to grow roughly proportionally to $L$, for fixed $\delta$. Thus $\bar{s}(\delta)$ can be made identically 1 in such a space.

Let $X$ denote the product $\mathbf{R} \times \mathbf{R}$ with the sup metric. Then even $s(0, L)$ in this space is at least $L / 2$, as one can see by considering $x=(0,0), y=(L, 0)$ and $z=(L / 2, L / 2)$. Thus the growth rate $\bar{s} \equiv 1$, as in the case of positive curvature.

Let $X, Y$ be two length spaces with instability functions $s_{X}, s_{Y}$. If $f: X \rightarrow Y$ is a map such that $\left|d_{X}-d_{Y} \circ f\right| \leq c$, it is easy to show that

$$
s_{X}(\delta, L) \leq 3 c+4 s_{Y}(\delta+3 c, L+c),
$$

and vice versa if $f$ is invertible. Applying this to the homeomorphism $\Pi$ of theorem 6.1 , we may conclude that $\bar{s}_{\mathcal{T}(S)}(\delta)=1$ for all $\delta>0$. This is the sense in which we say that $\mathcal{T}(S)$ is positively curved in the large. 


\section{References}

1. W. Abikoff, The real-analytic theory of Teichmüller space, Springer-Verlag, 1980, Lecture Notes in Mathematics no. 820.

2. L. Ahlfors, Conformal invariants: topics in geometric function theory, McGraw-Hill, 1973.

3. A. F. Beardon, The geometry of discrete groups, Springer-Verlag, 1983.

4. L. Bers, Spaces of degenerating Riemann surfaces, Discontinuous groups and Riemann surfaces, Ann. of Math. Stud. 79, Princeton Univ. Press, 1974, pp. 43-59.

5. J. Cannon, The theory of negatively curved spaces and groups, Ergodic theory, symbolic dynamics, and hyperbolic spaces (Trieste, 1989), Oxford Univ. Press, 1991, pp. 315-369.

6. M. Coornaert, T. Delzant, and A. Papadopoulos, Géométrie et theorie de groupes: les groups hyperboliques de Gromov, Springer-Verlag, 1990.

7. C. J. Earle and A. Marden, Geometric complex coordinates for Teichmüller space, manuscript.

8. A. Fathi, F. Laudenbach, and V. Poenaru, Travaux de Thurston sur les surfaces, vol. 66-67, Asterisque, 1979.

9. W. Fenchel and J. Nielsen, Discontinuous groups of non-Euclidean motions, unpublished manuscript.

10. R. Fricke and F. Klein, Vorlesungen über die Theorie der automorphen Funktionen, Teubner, Leipzig, 1897, 1912.

11. M. Gromov, Hyperbolic groups, Essays in Group Theory (S. M. Gersten, editor), MSRI Publications no. 8, Springer-Verlag, 1987.

12. S. Kerckhoff, The asymptotic geometry of Teichmüller space, Topology 19 (1980), 2341.

13. I. Kra, Horocyclic coordinates for Riemann surfaces and moduli space. I: Teichmüller and Riemann spaces of Kleinian groups, J. Amer. Math. Soc. 3 (1990), no. 3, 499-578.

14. B. Maskit, Decomposition of certain Kleinian groups, Acta Math. 130 (1973), 243-263.

15. Moduli of marked Riemann surfaces, Bull. Amer. Math. Soc. 80 (1974), 773777.

16. Comparison of hyperbolic and extremal lengths, Ann. Acad. Sci. Fenn. 10 (1985), 381-386.

17. H. A. Masur, On a class of geodesics in Teichmüller space, Ann. of Math. 102 (1975), 205-221.

18. The extension of the Weil-Petersson metric to the boundary of Teichmüller space, Duke Math. J. 43 (1976), 623-635.

19. - Transitivity properties of the horocyclic and geodesic flows on moduli space, J. D'analyse Math. 39 (1981), 1-10.

20. _ Two boundaries of Teichmüller space, Duke Math. J. 49 (1982), no. 1, 183-190.

21. H. A. Masur and M. Wolf, Teichmüller space is not Gromov hyperbolic, MSRI preprint No. 011-94, 1994.

22. Y. Minsky, Harmonic maps, length and energy in Teichmüller space, J. of Diff. Geom. 35 (1992), 151-217.

23. T_ Teichmüller geodesics and ends of hyperbolic 3-manifolds, Topology 32 (1993), 625-647.

24. W. Thurston, Three-Dimensional Geometry and Topology, Draft copy, University of Minnesota Geometry Center preprint, 1990.

25. S. A. Wolpert, On the Weil-Petersson geometry of the moduli space of curves, Amer. 
J. of Math. 107 (1985), 969-997.

26. _ Cut and paste deformation of Riemann surfaces, Ann. Acad. Sci. Fenn. 13 (1988), 401-413.

27. $\quad$ The hyperbolic metric and the geometry of the universal curve, J. Differential Geom. 31 (1990), 417-472.

Mathematics Department and IMS, SUNY Stony Brook, NY 11794

E-mail address: yair@math.sunysb.edu 